\title{
Heat and mass transfer and thermodynamic analysis of power-law fluid flow in a porous microchannel
}

\author{
Mahnaz Javidi Sarafan ${ }^{1} \cdot$ Rasool Alizadeh $^{1} \cdot$ Abolfazl Fattahi $^{2} \cdot$ Mostafa Valizadeh Ardalan $^{3} \cdot$ Nader Karimi $^{4}$
}

Received: 25 March 2020 / Accepted: 7 April 2020 / Published online: 24 April 2020

(c) The Author(s) 2020

\begin{abstract}
Transfer of heat and mass and thermodynamic irreversibilities are investigated in a porous, parallel-plate microreactor in which the working fluid is non-Newtonian. The investigated microreactor features thick flat walls with uneven thicknesses, which can be subject to different thermal loads. The dimensionless governing equations of the resultant asymmetric problem are first derived theoretically and then solved numerically by using a finite volume technique. This results in two-dimensional solutions for the velocity, temperature and concentration fields as well as the distributions of Nusselt number and local and total entropy generations. The results clearly demonstrate the significance of the numerical value of the power-law index and departure from Newtonian behavior of the fluid. In particular, it is shown that by increasing the value of power-law index the Nusselt number on the wall decreases. This leads to the intensification of the temperature gradients in the system and therefore magnifies the local and total entropy generations. Also, it is shown that the wall thickness and thermal asymmetry can majorly affect the heat transfer process and thermodynamic irreversibility of the microreactor. It is noted that the current work is the first comprehensive study of heat transfer and entropy generation in porous micro-chemical reactor with non-Newtonian, power-law fluid.
\end{abstract}

Keywords Porous microchannel · Microreactor · Non-equilibrium thermodynamics · Entropy generation · Power-law fluid · Forced convection

\section{List of symbols \\ $a_{\mathrm{sf}} \quad$ Interfacial area per unit volume of porous media $\left(\mathrm{m}^{-1}\right)$ \\ Bi Biot number \\ C Mass species concentration $\left(\mathrm{kg} \mathrm{m}^{-3}\right)$ \\ $C_{0} \quad$ Inlet concentration $\left(\mathrm{kg} \mathrm{m}^{-3}\right)$ \\ $C_{\mathrm{p}} \quad$ Specific heat capacity $\left(\mathrm{J} \mathrm{K}^{-1} \mathrm{~kg}^{-1}\right)$ \\ $D \quad$ Mass diffusion coefficient $\left(\mathrm{m}^{-2} \mathrm{~s}^{-1}\right)$ \\ Da Darcy number \\ $D_{\mathrm{T}} \quad$ Coefficient of thermal mass diffusion, $\left(\mathrm{m}\left(\mathrm{K} \mathrm{kg} \mathrm{s}^{-1}\right)\right.$}

Nader Karimi

Nader.Karimi@glasgow.ac.uk

1 Department of Mechanical Engineering, Quchan Branch, Islamic Azad University, Quchan, Iran

2 Department of Mechanical Engineering, University of Kashan, Kashan, Iran

3 Department of Mechanical Engineering, Shahroud University of Technology, Shahroud, Iran

4 School of Engineering, University of Glasgow, Glasgow G12 8QQ, UK $h_{1} \quad$ Half-thickness of the microchannel (m)

$h_{2} \quad$ Half-height of microchannel (m)

$h_{\text {sf }} \quad$ Interstitial heat transfer coefficient $\left(\mathrm{W} \mathrm{K}^{-1} \mathrm{~m}^{-2}\right)$

$H_{\mathrm{w}} \quad$ Wall heat transfer coefficient $\left(\mathrm{W} \mathrm{K}^{-1} \mathrm{~m}^{-2}\right.$ )

$k \quad$ Effective thermal conductivity ratio of the fluid and the porous solid

$k_{1} \quad$ Thermal conductivity of wall $1\left(\mathrm{~W} \mathrm{~K}^{-1} \mathrm{~m}^{-2}\right)$

$k_{2} \quad$ Thermal conductivity of wall $2\left(\mathrm{~W} \mathrm{~K}^{-1} \mathrm{~m}^{-2}\right)$

$k_{\mathrm{e} 1} \quad$ Ratio of thermal conductivity of wall 1 and thermal conductivity of the porous solid

$k_{\mathrm{e} 2} \quad$ Ratio of thermal conductivity of wall 2 and thermal conductivity of the porous solid

$k_{\text {ef }} \quad$ Effective thermal conductivity of the fluid phase (W K $\mathrm{K}^{-1} \mathrm{~m}^{-2}$ )

$k_{\text {es }} \quad$ Effective thermal conductivity of the solid phase of the porous medium $\left(\mathrm{W} \mathrm{K}^{-1} \mathrm{~m}^{-2}\right.$ )

$k_{\mathrm{R}} \quad$ Reaction rate constant on the walls $\left(\mathrm{m} \mathrm{s}^{-1}\right)$

$L \quad$ Length of the microchannel (m)

$m \quad$ Power law consistency index

$n \quad$ Power law index

$N_{\text {DI }} \quad$ Dimensionless diffusive irreversibility

$N_{\mathrm{FF}} \quad$ Dimensionless fluid friction irreversibility 


\begin{tabular}{|c|c|}
\hline$N_{\text {int }}$ & $\begin{array}{l}\text { Dimensionless interstitial heat transfer } \\
\text { irreversibility }\end{array}$ \\
\hline$N_{\mathrm{f}}$ & Dimensionless fluid and interstitial irreversibility \\
\hline$N_{\text {f,ht }}$ & Dimensionless fluid heat transfer irreversibility \\
\hline$N_{\mathrm{s}}$ & $\begin{array}{l}\text { Dimensionless porous solid and interstitial } \\
\text { irreversibility }\end{array}$ \\
\hline$N_{\mathrm{s}, \mathrm{ht}}$ & Dimensionless heat transfer irreversibility \\
\hline$N_{\mathrm{w} 1}$ & Dimensionless lower wall irreversibility \\
\hline$N_{\mathrm{w} 2}$ & Dimensionless upper wall irreversibility \\
\hline$N_{\mathrm{pm}}$ & Dimensionless total porous medium irreversibility \\
\hline$N_{\text {Tot }}$ & Dimensionless total entropy \\
\hline $\mathrm{Nu}$ & Nusselt number \\
\hline$p$ & Pressure $(\mathrm{Pa})$ \\
\hline $\mathrm{Pe}$ & Peclet number \\
\hline$X$ & Dimensionless axial coordinate \\
\hline $\operatorname{Pr}$ & Prandtl number \\
\hline$Q$ & Wall heat flux ratio \\
\hline$q_{1}^{\prime \prime}$ & Lower wall heat flux $\left(\mathrm{W} \mathrm{m}^{-2}\right)$ \\
\hline$q_{2}^{\prime \prime}$ & Upper wall heat flux $\left(\mathrm{W} \mathrm{m}^{-2}\right)$ \\
\hline $\mathrm{Re}$ & Reynolds number on the basis of channel height \\
\hline$R$ & Specific gas constant $\left(\mathrm{J} \mathrm{K}^{-1} \mathrm{~kg}^{-1}\right)$ \\
\hline$S$ & Shape factor of the porous medium \\
\hline$\dot{S}_{\mathrm{DI}}^{\prime \prime \prime}$ & $\begin{array}{l}\text { Volumetric entropy generation due to mass diffu- } \\
\text { sion }\left(\mathrm{W} \mathrm{K}^{-1} \mathrm{~m}^{-3}\right)\end{array}$ \\
\hline$\dot{S}_{\mathrm{f}}^{\prime \prime \prime}$ & $\begin{array}{l}\text { Volumetric entropy generation in the fluid } \\
\left(\mathrm{W} \mathrm{K}^{-1} \mathrm{~m}^{-3}\right)\end{array}$ \\
\hline$\dot{S}_{\mathrm{s}}^{\prime \prime \prime}$ & $\begin{array}{l}\text { Volumetric entropy generation in the porous solid } \\
\left(\mathrm{W} \mathrm{K}^{-1} \mathrm{~m}^{-3}\right)\end{array}$ \\
\hline$\dot{S}_{\mathrm{w} 1}^{\prime}$ & $\begin{array}{l}\text { Volumetric entropy generation rate from lower wall } \\
\left(\mathrm{W} \mathrm{K}^{-1} \mathrm{~m}^{-3}\right)\end{array}$ \\
\hline$\dot{S}_{\mathrm{w} 2}^{\prime}$ & $\begin{array}{l}\text { Volumetric entropy generation rate from upper wall } \\
\left(\mathrm{W} \mathrm{K}^{-1} \mathrm{~m}^{-3}\right)\end{array}$ \\
\hline $\mathrm{Sr}$ & Soret number \\
\hline$T$ & Temperature (K) \\
\hline$u$ & Fluid velocity $\left(\mathrm{m} \mathrm{s}^{-1}\right)$ \\
\hline $\bar{u}$ & Average velocity over cross-section $\left(\mathrm{m} \mathrm{s}^{-1}\right)$ \\
\hline$Y$ & Dimensionless transverse coordinate \\
\hline$X$ & Dimensionless axial coordinate \\
\hline$y$ & Dimensional transverse coordinate \\
\hline$x$ & Dimensional axial coordinate \\
\hline
\end{tabular}

\section{Greek symbols}

$\mu \quad$ Dynamic viscosity $\left(\mathrm{N} \mathrm{s} \mathrm{m}^{-2}\right)$

$\kappa \quad$ Permeability $\left(\mathrm{m}^{2}\right)$

$\rho \quad$ Density $\left(\mathrm{kg} \mathrm{m}^{-3}\right)$

$\theta \quad$ Dimensionless temperature

$\phi \quad$ Dimensionless concentration

$\xi \quad$ Aspect ratio of the microchannel

$\varepsilon \quad$ Porosity of the porous medium

$\gamma \quad$ Damköhler number

$\omega \quad$ Dimensionless heat flux

$\varphi \quad$ Irreversibility distribution ratio
Subscripts
w1 Of the lower wall surface
w2 Of the upper wall surface
f Of fluid
s Of porous solid
1 Of lower wall
2 Of upper wall

\section{Introduction}

Catalytic porous microchannels, where catalytic channels of small sizes filled with porous media, have received increased interest in traditional and advanced chemical and energy technologies in the last decade. Featuring high surface-tovolume ratios, catalytic porous microchannels can readily achieve the desired performance on transport and chemical reactions [1-3]. Therefore, extensive studies have been reported in recent years on applications of catalytic porous microchannels [4-7]. However, due to the relatively complex natures in catalytic porous microchannels, our understanding of relevant fundamental mechanisms is still far from satisfactory and there are considerable voids for efficient utilization of catalytic porous microchannels. Thus, accurately quantifying the inherent characteristics in catalytic porous microchannels is the prerequisite for their subsequent efficient utilization and optimization. Among the massive aspects that require in-depth investigation for catalytic porous microchannels, transport processes and entropy generation are of vital importance as they are directly related to the overall performance of a catalytic porous microchannel.

Owing to the coupled complexities of multi-physics and geometrical topology in a typical catalytic porous microchannel, a comprehensive investigation of intrinsic characteristics and engineering principles through experiments may be too expensive [8-10]. Additionally, accurate measurements at the small spatiotemporal scales in catalytic porous microchannels pose great challenges to current measuring techniques $[11,12]$. Considering the dramatical increase in computational power and rapid development of theoretical models, numerical modeling has played greater roles in scientific research and engineering optimization for catalytic porous microchannels. Although the general transport [13-15] and entropy generation [16-18] characteristics of microreactors have been investigated to some extent, their behavior under non-Newtonian fluids is still largely unknown. As a matter of fact, there is still a significant knowledge gap in understating convective heat and mass transfer in porous media and microchannels with nonNewtonian fluid flow [19-21]. In the following, some of the recent studies in these areas are briefly discussed.

Neffah et al. [22] conducted a numerical study of heat and mass transfer in a non-Newtonian fluid in a parallel-plate 
channel partly filled with an anisotropic porous medium. They investigated the effects of various parameters pertinent to the porous medium, the non-Newtonian fluid and the chemical reaction on the velocity, temperature and concentration distributions, as well as mean Nusselt and Sherwood numbers. Neffah et al. [22] showed that compared with the isotropic case, the anisotropy of a porous medium can lead to significant improvements in heat and mass transfer. The shear-thickening fluids exhibit the highest values of mean Nusselt and Sherwood numbers at large Darcy number values. The work of Neffah et al. [22] clarified that an increment in the chemical reaction parameters mitigates heat and mass transfer rates.

In a recent numerical work, Wang et al. [23] investigated entropy generation in thermal natural convection with differentially discrete heat boundary conditions at various Rayleigh numbers. This study showed that the thermal, viscous and total entropy generation increase with the increase in Rayleigh number. It also confirmed that the system's availability improves in the presence of effective heat source at the bottom of the boundary.

Saeed et al. [24] analyzed the methods of biofuel processing in diesel engines to evaluate non-Newtonian biofuel flow in a magnetic microreactor. It was shown that variations in the magnetic field strength and the thermo-physical properties of the fluid could cause considerable temperature differences. It was also observed that the alterations in the properties of Casson fluid as well as the change in the magnitude and angle of the magnetic field could affect Nusselt number. It was further found that the extent of the variations is strongly dependent on the wall thickness.

Gholamalizadeh et al. [25] performed a two-dimensional numerical study of a convective flow in a nanofluid water/ FMWCNT-coated microchannel. The slip velocity boundary condition was applied for the solid walls. The results showed that as compared to Reynolds number, Darcy number has a more pronounced effect upon the velocity profile. It was also found that increasing porosity did not affect the velocity profile growth in any way.

It has been already reported that working fluids have significant impact on the overall performance of a catalytic porous microchannel $[1,2,7]$. In a numerical study, Maleki et al. [26] investigated flow and heat transfer in non-Newtonian nanofluids over porous surfaces. They investigated the effects of parameters, such as power-law index, volume fraction of nanoparticles, nanoparticles type and permeability parameter on the flow and heat transfer of the desired nanofluid. The study found that the hydro-thermal properties of non-Newtonian nanofluid flows exhibited different behaviors as compared with the common working fluids. For instant, the injections and permeability in the plate showed higher heat transfer performance for non-Newtonian nanofluids in comparison with Newtonian nanofluids.
In their numerical work, Animasaun and Pop [27] considered the geometry and position of the surface for the study of the desired fluid flow in order to evaluate the flow performance on the target parameters. The results showed that the non-Newtonian fluid temperature distribution was higher than that of the Newtonian fluid. It was also found that by applying the non-Newtonian fluid, the rate of local heat transfer decreases more rapidly. The apparent disagreement of this finding with that reported by Maleki et al. [26] is a clear indication that the field of heat convection in nonNewtonian fluids has not settled yet.

Al-Rashed et al. [28] conducted a study to evaluate the thermal and entropic properties of a non-Newtonian nanofluid containing $\mathrm{CuO}$ particles in the substrate (MCHS). This article discusses the effects of concentration of nanoparticles, the Reynolds number as well as the geometric size intended for MCHS based on the first and second laws of thermodynamics. The results showed that increasing Reynolds number improves the performance of MCHS by increasing the fluid convection heat transfer coefficient of the working fluid, which in turn results in uniform temperature of the substrate. They further revealed that the use of nonNewtonian nanofluid instead of the base ordinary fluid leads to an increase in heat transfer efficiency against increasing the pressure drop. Also, by using non-Newtonian nanofluid, the least amount of entropy generation in the system can be achieved.

So far, most studies and applications of catalytic porous microchannels have used Newtonian fluids, while effects of non-Newtonian fluids on thermal transport and entropy generation have been seldomly touched [29, 30]. However, non-Newtonian fluids are more common in practice and our knowledge of their influences on performance of catalytic porous microchannels should be advanced. To partially close the gap of clarifying the effects of non-Newtonian fluids, a catalytic porous microchannel with power-law fluids has been studied in this study. The reasons to choose powerlaw fluids in this work are twofold. First, the mathematical description of power-law fluids is relatively simple, which can greatly benefit the formulation of conservation equations for modeling of thermal transport and entropy generation in porous microchannels. Second, as a widely encountered type of non-Newtonian fluids, power-law fluids are rather representative in the engineering.

In this work, numerical analysis of thermal transport and entropy generation of a typical catalytic porous microchannel filled with power-law fluids has been conducted. To focus on the effects of power-law fluids, catalytic reactions only happen at the sidewalls of channels and Soret effect is taken into consideration. In the following, governing equations of the studied system are described and discussed. Then, analytical solutions of relevant governing equations are derived and solved numerically. After validating the 
developed theoretical solution, the differences of thermal transport and entropy generation between Newtonian and power-law fluids are compared and discussed. Finally, a comprehensive parametric analysis was conducted to study the effects of physical properties of power-law fluids.

\section{Theoretical and numerical methods}

\section{Assumptions}

The assumptions considered in this study are presented as the following.

- The flow is laminar, steady and hydrodynamically fully developed.

- The fluid is non-Newtonian power law.

- The flow regime is assumed to be continuous with Knudsen number lower than 0.3 .

- All chemical reactions on the catalyst are assumed to be of zeroth order.

- The effect of temperature on the surface reactions is neglected.

- The porous medium is isotropic, homogeneous, saturated with fluid and under thermally non-equilibrium conditions (LTNE).

\section{Governing equations and boundary conditions}

The rheological equation for an isotropic, incompressible flow of a power-law fluid is [31]

$\mu=m\left|\frac{\partial u}{\partial y}\right|^{\mathrm{n}-1}$,

where $\mathrm{m}, u, y$ and $n$ are the power-law consistency index, fluid velocity, dimensional transverse coordinate and powerlaw behavior index, respectively.

The Darcy-Brinkman equation for the momentum equation $[2,32,33]$ is considered as the follows:

$-\frac{\partial p}{\partial x}+\mu_{\mathrm{eff}} \frac{\partial u^{2}}{\partial y^{2}}-\frac{\mu}{\kappa} u=0 \quad-h_{1} \leq y<h_{1}$.

The following equations represent the energy transfer in the solid and fluid phases:

$k_{2} \frac{\partial}{\partial y}\left[\frac{\partial T_{2}}{\partial y}\right]=0, \quad h_{1}<y \leq h_{2}$

$k_{\mathrm{ef}} \frac{\partial^{2} T_{\mathrm{f}}}{\partial y^{2}}+h_{\mathrm{sf}} a_{\mathrm{sf}}\left(T_{\mathrm{s}}-T_{\mathrm{f}}\right)=\rho_{\mathrm{f}} C_{\mathrm{p}, \mathrm{f}} u \frac{\partial T_{\mathrm{f}}}{\partial x}, \quad-h_{1} \leq y<h_{1}$ $k_{\mathrm{es}} \frac{\partial^{2} T_{\mathrm{s}}}{\partial y^{2}}-h_{\mathrm{sf}} a_{\mathrm{sf}}\left(T_{\mathrm{s}}-T_{\mathrm{f}}\right)=0, \quad-h_{1} \leq y<h_{1}$

$k_{1} \frac{\partial}{\partial y}\left[\frac{\partial T_{1}}{\partial y}\right]=0 \quad-h_{2} \leq y<-h_{1}$.

The mass transfer involves the effect of thermal diffusion (Sorter effects) on the Fickian diffusion in the advective-diffusive system, as follows [1]:

$u \frac{\partial C}{\partial x}=D \frac{\partial^{2} C}{\partial y^{2}}-D_{\mathrm{T}} \frac{\partial^{2} T_{\mathrm{f}}}{\partial y^{2}} \quad-h_{1} \leq y<h_{1}$.

In Eqs. (2)-(4), $p$ is thepressure, $x$ is the dimensional axial coordinate, $\mu$ and $\mu_{\text {eff }}$ are the viscosity of the fluid and the effective viscosity of the porous medium, respectively, $\kappa$ is the permeability of the porous insert, $h_{1}$ and $h_{2}$, refer to the half-thickness and half-height of the microchannel. Further, $k_{\text {ef }}$ and $k_{\text {es }}$ are the effective thermal conductivity of the fluid phase and solid phase of the porous medium, $k_{1}$ and $k_{2}$ are the thermal conductivity of wall 1 and wall 2 (see Fig. 1), $h_{\mathrm{sf}}$ and $a_{s f}$ are the interstitial heat transfer coefficient and interfacial area per unit volume of porous media. Furthermore,
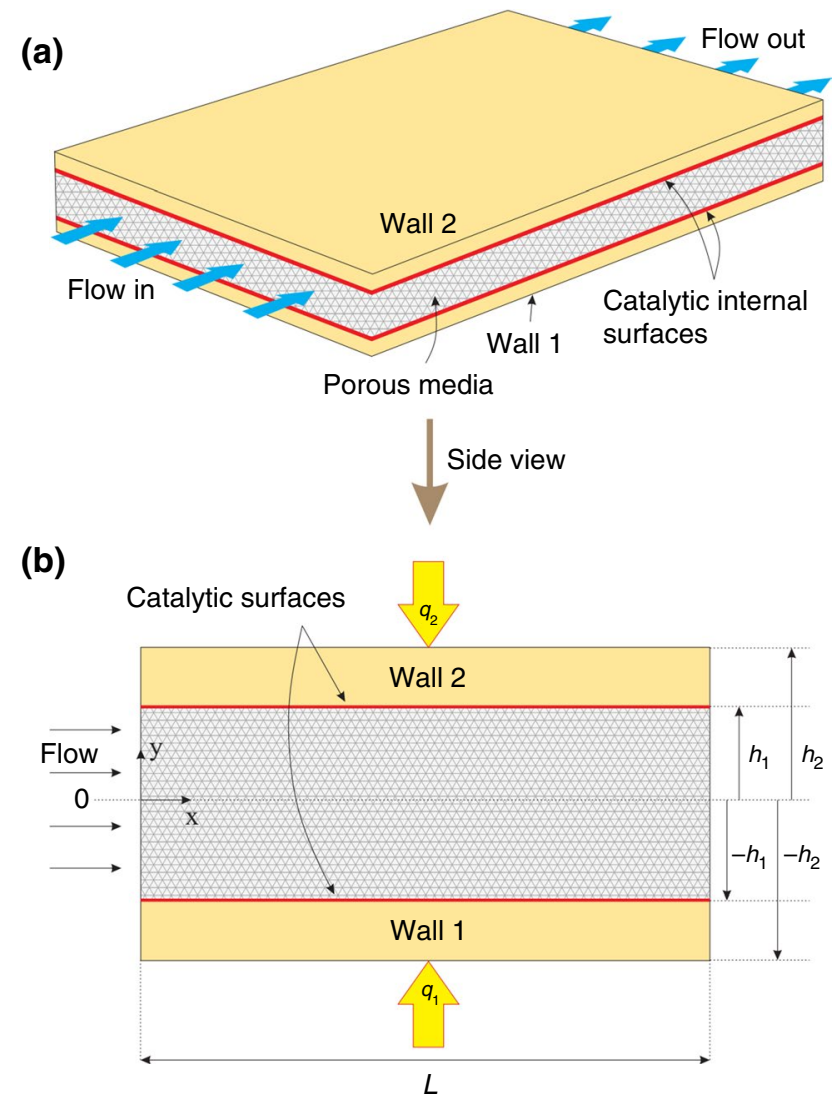

Fig. 1 Schematic a isometric and $\mathbf{b}$ side view of the porous microchannel under investigation 
$T_{\mathrm{s}}, T_{\mathrm{f}}, T_{1}$ and $T_{2}$ denote the temperature of solid phase, fluid phase, lower and upper wall, respectively. Also, $\rho_{\mathrm{f}}$ and $C_{\mathrm{p}, \mathrm{f}}$ show the density and specific heat capacity of the fluid, $C$ is the mass species concentration, $D$ denotes the effective mass diffusion coefficient and $D_{T}$ represents the coefficient of thermal mass diffusion.

Although most solutes feature positive coefficients of thermal mass diffusion representing that the mass can be moved down the thermal gradient, the other have the negative coefficients and are able to be diffused up the thermal gradient. Therefore, the negative or positive sign of the last term in Eq. (4) is acceptable.

For energy and momentum equations, the boundary conditions are

$y=h_{2}:\left.k_{2} \frac{\partial T_{2}}{\partial y}\right|_{y=h_{2}}=q_{2}^{\prime \prime}$,

$y=h_{1}: u_{\mathrm{f}}=0, T_{2}=T_{\mathrm{f}}=T_{\mathrm{s}}=T_{\mathrm{w} 2}, q_{2}^{\prime \prime}=\left.k_{\mathrm{ef}} \frac{\partial T_{\mathrm{f}}}{\partial y}\right|_{y=h_{1}}+\left.k_{\mathrm{es}} \frac{\partial T_{\mathrm{s}}}{\partial y}\right|_{y=h_{1}}$

$y=0: \frac{\mathrm{d} u_{\mathrm{f}}}{\mathrm{d} y}=0$

$y=-h_{1}: u_{\mathrm{f}}=0, T_{2}=T_{\mathrm{f}}=T_{\mathrm{s}}=T_{\mathrm{w} 1}$,

$q_{1}^{\prime \prime}=-\left.k_{\mathrm{ef}} \frac{\partial T_{\mathrm{f}}}{\partial y}\right|_{y=-h_{1}}-\left.k_{\mathrm{es}} \frac{\partial T_{\mathrm{s}}}{\partial y}\right|_{y=-h_{1}}$

$y=-h_{2}:\left.k_{1} \frac{\partial T_{1}}{\partial y}\right|_{y=-h_{2}}=q_{1}^{\prime \prime}$

The concentration boundary conditions are expressed as follows:

$y=-h_{1}: C=C_{0}$

$y=0: D \frac{\partial C}{\partial y}=D_{\mathrm{T}} \frac{\partial T_{\mathrm{f}}}{\partial y}$.

Next, the dimensionless parameters for better physical analysis are stated in Table 1.
The dimensionless temperature follows the given chain, $\mathrm{i}=1,2, \mathrm{~s}, \mathrm{f}$.

In Table $1, q_{1}^{\prime \prime}$ and $q_{1}^{\prime \prime}$ are the lower and upper wall heat flux, $\theta$ is the dimensionless temperature, $k_{\mathrm{R}}$ is the reaction rate constant on the walls, $X$ and $Y$ are the dimensionless axial and transverse coordinates, $\mathrm{Da}, B i, \gamma, \mathrm{Sr}, \mathrm{Pr}, \mathrm{Re}$ and $\mathrm{Pe}$ are the Darcy, Biot, Damköhler, Soret, Prandtl, Reynolds and Peclet numbers, respectively. Also, $Q$ represents the wall heat flux ratio, $S$ is the shape factor of the porous medium, $\xi$ shows the aspect ratio of the microchannel, $\varepsilon$ is the porosity of the porous medium, $\phi$ and $U$ denote the dimensionless concentration and fluid velocity, respectively. Further, $k_{\mathrm{e} 1}$ is the ratio of thermal conductivity of wall 1 and thermal conductivity of the porous solid, $k_{\mathrm{e} 2}$ is the ratio of thermal conductivity of wall 2 and thermal conductivity of the porous solid and $k$ refers to the effective thermal conductivity ratio of the fluid and the porous solid.

\section{Analytical derivation of the dimensionless governing equations}

Given the dimensionless equations defined in Table 1, Eq. (2) can be expressed as

$n \frac{\mathrm{d}^{2} U}{\mathrm{~d} Y^{2}}-\frac{U}{\mathrm{Da}}+1=0 \quad-Y_{1} \leq Y<Y_{1}$.

Similarly, no-slip boundary conditions and axial symmetry conditions at $Y=0$ can be expressed as follows:

$U\left( \pm Y_{1}\right)=0$,

$U^{\prime}(0)=0$.

Solution of Eq. (7) can be expressed with

$U=\mathrm{Da}\left(1-\frac{\operatorname{Cosh}(S Y)}{\operatorname{Cosh}\left(S Y_{1}\right)}\right) \quad-Y_{1} \leq Y<Y_{1}$.

Using Eq. (8), the average dimensionless velocity across the channel equals

$\bar{u}=\mathrm{Da}\left(1-\frac{\tanh \left(S Y_{1}\right)}{S Y_{1}}\right)$.
Table 1 Dimensionless parameters

\begin{tabular}{lllll}
\hline$B i=\frac{h_{\mathrm{sf}} a_{\mathrm{sf}} h_{2}^{2}}{k_{\mathrm{es}}}$ & $S r=\frac{\left(q_{1}^{\prime \prime}+q_{2}^{\prime \prime}\right) h_{1} D_{\mathrm{T}}}{2 C_{0} k_{\mathrm{f}} D}$ & $\operatorname{Pr}=\frac{C_{\mathrm{p}, \mathrm{f}} \cdot m}{k_{\mathrm{ef}}}\left(\frac{\bar{u}}{h_{2}}\right)^{n-1}$ & $\operatorname{Re}=\frac{\rho \bar{u}^{2-n}\left(2 h_{2}\right)^{n}}{m}$ & $\operatorname{Pe}=\frac{\bar{u} h_{2}}{D}$ \\
\hline $\mathrm{Da}=\frac{\kappa}{h_{2}^{2}}$ & $\gamma=\frac{k_{\mathrm{R}} h_{1}}{D}$ & $S=\frac{1}{\sqrt{n \cdot \mathrm{Da}}}$ & $\theta_{i}=\frac{2\left(T_{i}-T_{\mathrm{w}, \mathrm{in}}\right) k_{\mathrm{es}}}{\left(q_{1}^{\prime \prime}+q_{1}^{\prime \prime}\right) h_{2}}$ & $Q=\left(\frac{q_{2}^{\prime \prime}}{q_{1}^{\prime \prime}+q_{2}^{\prime \prime}}\right)$ \\
$Y=\frac{y}{h_{2}}$ & $Y_{1}=\frac{h_{1}}{h_{2}}$ & $X=\frac{x}{L}$ & $\xi=\frac{h_{2}}{L}$ & $U=\frac{u}{u_{\mathrm{r}}}$ \\
$k_{\mathrm{e} 1}=\frac{k_{1}}{k_{\mathrm{es}}}$ & $k_{\mathrm{e} 2}=\frac{k_{2}}{k_{\mathrm{es}}}$ & $k=\frac{k_{\mathrm{ef}}}{k_{\mathrm{es}}}=\frac{\varepsilon k_{\mathrm{f}}}{(1-\varepsilon) k_{\mathrm{s}}}$ & $\phi=\frac{C}{C_{0}}$ & $u_{\mathrm{r}}=-\frac{h_{2}^{2}}{\mu_{\mathrm{f}}} \frac{\partial p_{\mathrm{f}}}{\partial x}$
\end{tabular}


By combining Eqs. (9) and (10), the following ratio of absolute and mean is defined in Eq. (11):

$u / \bar{u}=U / \bar{U}=\frac{S Y_{1}\left(\operatorname{Cosh}\left(S Y_{1}\right)-\operatorname{Cosh}(S Y)\right)}{S Y_{1} \operatorname{Cosh}\left(S Y_{1}\right)-\operatorname{Sinh}\left(S Y_{1}\right)}$.

Assuming the flow to be fully developed, as given the assumptions in Section "Assumptions", the conditions stated are $[1,32]$

$\frac{\partial T_{\mathrm{f}}}{\partial x}=\frac{\partial \bar{T}_{\mathrm{f}}}{\partial x}=\frac{\partial T_{\mathrm{s}}}{\partial x}=\frac{\partial \bar{T}_{\mathrm{s}}}{\partial x}=\frac{\partial T_{\mathrm{w} 1}}{\partial x}=\frac{\partial T_{\mathrm{w} 2}}{\partial x}=\Omega=$ Constant.

Subsequently, the temperature equation is [1]

$T_{\mathrm{i}}(x, y)=f_{\mathrm{i}}(y)+\Omega x \quad i=1,2, s, f$.

In Eq. (13), the function $f_{\mathrm{i}}(y)$ is obtained by solving Eq. (3a) with the resultant equation from combination of Eq. (3b) and (3c). By placing the heat flux boundary conditions in Eq. (5b) and (5d), we have

$q_{1}^{\prime \prime}+q_{2}^{\prime \prime}=\rho_{\mathrm{f}} C_{\mathrm{p}, \mathrm{f}} \int_{-h_{1}}^{h_{1}} u \frac{\partial T_{\mathrm{f}}}{\partial x} \mathrm{~d} y$.

Substituting the velocity from Eq. (11) into Eq. (14) and using the dimensionless parameters defined in Table 1, the following equation is taken:

$\frac{\partial \bar{T}_{\mathrm{f}}}{\partial x}=\frac{1}{2 \rho_{\mathrm{f}} C_{\mathrm{p}, \mathrm{f}} \bar{u} h_{1} h_{2}}\left[h_{1} q_{1}^{\prime \prime}+h_{2} q_{2}^{\prime \prime}\right]=\Omega$,

where the average non-Newtonian fluid temperature is given as follows:

$\bar{T}_{\mathrm{f}}=\frac{1}{2 \bar{u} h_{1}} \int_{-h_{1}}^{h_{1}} u T_{\mathrm{f}} \mathrm{d} y$.

Using the dimensionless parameters of Table 1 and rearranging Eqs. (11) to (15), the dimensionless form of Eq. (3a) to $(3 \mathrm{~d})$ is obtained.

$k_{\mathrm{e} 2} \theta_{2}^{\prime \prime}=0, Y_{1}<Y \leq 1$

$k \theta_{\mathrm{f}}^{\prime \prime}+\operatorname{Bi}\left(\theta_{\mathrm{s}}-\theta_{\mathrm{f}}\right)+D_{3} \operatorname{Cosh}(S Y)+D_{4}=0,-Y_{1} \leq Y<Y_{1}$

$\theta_{\mathrm{s}}^{\prime \prime}-B i\left(\theta_{\mathrm{s}}-\theta_{\mathrm{f}}\right)=0,-Y_{1} \leq Y<Y_{1}$

$k_{\mathrm{e} 1} \theta_{1}^{\prime \prime}=0 .-1 \leq Y<-Y_{1}$

Algebraic manipulating Eq. (17b) and (17c) gives $k \theta_{\mathrm{f}}^{\prime \prime \prime \prime}-B i(1+k) \theta_{\mathrm{f}}^{\prime \prime \prime \prime}+\left(S^{2}-B i\right) D_{3} \operatorname{Cosh}(S Y)-\mathrm{Bi} D_{4}=0$,

$-Y_{1} \leq Y<Y_{1}$

$k \theta_{\mathrm{s}}^{\prime \prime \prime \prime}-\operatorname{Bi}(1+k) \theta_{\mathrm{s}}^{\prime \prime}-\operatorname{Bi}\left(D_{3} \operatorname{Cosh}(S Y)+D_{4}\right)=0 \quad-Y_{1} \leq Y<Y_{1}$.

The boundary conditions of Eq. (17a-17d) and Eq. (18a) and (18b) are expressed by the following relations.

$\theta_{\mathrm{f}}\left(-Y_{1}\right)=\theta_{\mathrm{s}}\left(-Y_{1}\right)=0, \theta_{\mathrm{f}}\left(-Y_{1}\right)=\theta_{\mathrm{s}}\left(-Y_{1}\right)=\theta_{\mathrm{w} 2}$

$\theta_{\mathrm{s}}^{\prime \prime}\left(-Y_{1}\right)=\theta_{\mathrm{s}}^{\prime \prime}\left(Y_{1}\right)=0$,

$\theta_{\mathrm{f}}^{\prime \prime}\left(-Y_{1}\right)=\theta_{\mathrm{f}}^{\prime \prime}\left(Y_{1}\right)=-\frac{1}{k}\left(D_{3} \operatorname{Cosh}(S Y)+D_{4}\right)$,

$\theta_{1}\left(-Y_{1}\right)=0, k_{\mathrm{e} 1} \theta_{1} \prime(1)=2(1-Q)$,

$\theta_{2}\left(Y_{1}\right)=\theta_{\mathrm{w} 2}, k_{\mathrm{e} 2} \theta_{2}^{\prime}(1)=2 Q$,

By applying Eq. (19a) to (19h), it is possible to solve analytically the dimensionless cross-sectional temperature profiles using Eqs. (17a), (17b) and (18a), as the following:

$\theta_{2}(Y)=E_{1}+E_{2} Y, Y_{1}<Y \leq 1$,

$\theta_{\mathrm{f}}(Y)=E_{4} \operatorname{Cosh}(S Y)+E_{5} \operatorname{Cosh}(\alpha Y)+E_{6} Y^{2}$

$+E_{7} Y+E_{8},-Y_{1}<Y \leq Y_{1}$

$\theta_{\mathrm{s}}(Y)=E_{10} \operatorname{Cosh}(S Y)+E_{11} \operatorname{Cosh}(\alpha Y)+E_{6} Y^{2}$

$$
+E_{7} Y+E_{12},-Y_{1}<Y \leq Y_{1}
$$

$\theta_{1}(Y)=E_{13}+E_{14} Y .-1 \leq Y<-Y_{1}$

where alpha equals

$\alpha=\sqrt{B i\left(1+k^{-1}\right)}$.

By placing dimensionless cross-sectional temperature profiles of Eq. (20) inside Eq. (13), the final dimensionless temperature profiles are expressed by

$\theta_{2}(X, Y)=\frac{2 X}{\operatorname{Re} \operatorname{Pr} k Y_{1} \xi}+E_{1}+E_{2} Y, Y_{1}<Y \leq 1$

$$
\begin{aligned}
\theta_{\mathrm{f}}(X, Y)= & \frac{2 X}{\operatorname{Re} \operatorname{Pr} k Y_{1} \xi}+E_{4} \operatorname{Cosh}(S Y)+E_{5} \operatorname{Cosh}(\alpha Y) \\
& +E_{6} Y^{2}+E_{8}+E_{7} Y,-Y_{1} \leq Y<Y_{1}
\end{aligned}
$$


$\theta_{\mathrm{S}}(X, Y)=\frac{2 X}{\operatorname{Re} \operatorname{Pr} k Y_{1} \xi}+E_{10} \operatorname{Cosh}(S Y)+E_{11} \operatorname{Cosh}(\alpha Y)$ $+E_{6} Y^{2}+E_{12}+E_{7} Y,-Y_{1} \leq Y<Y_{1}$

$\theta_{1}(X, Y)=\frac{2 X}{\operatorname{Re} \operatorname{Pr} k Y_{1} \xi}+E_{13}+E_{14} Y .-1 \leq Y<Y_{1}$

The following equation is developed by applying Leibniz's rule [34] on Eq. (4):

$\left[\int_{0}^{h_{1}} u \cdot C \cdot \mathrm{d} y\right]+K_{\mathrm{R}} \mathrm{d} x-\left[\int_{0}^{h_{1}} u \cdot C \cdot \mathrm{d} y+\frac{\partial}{\partial x}\left(\int_{0}^{h_{1}} u \cdot C \cdot \mathrm{d} y\right) \mathrm{d} x\right]=0$.

The velocity profile along the microchannel is symmetri$\mathrm{cal}$, and therefore half of the channel is considered. The average velocity in half of the microchannel is given by

$\int_{0}^{h_{1}} u \cdot \frac{\partial C}{\partial x} \cdot \mathrm{d} y=K_{\mathrm{R}}$

The average flow velocity is defined as

$\bar{u}=\frac{1}{h_{1}} \int_{0}^{h_{1}} u \cdot \mathrm{d} y$.

By setting $C_{0}$ as the initial concentration, the following equation is revealed.

$\frac{\partial C}{\partial x}=\frac{K_{R}}{h_{1} \bar{u}}$.

Using the dimensionless parameters defined in Table 1, the concentration distribution yields

$C(x)=\frac{K_{\mathrm{R}} x}{h_{1} \bar{u}}$.

Replacing Eq. (26) into Eq. (4), the distribution of dimensionless concentration function is given as

$\phi(x)=\frac{\gamma X}{P e Y_{1} \xi}$.

Applying the dimensionless parameters of Table 1 and the ratio of velocity in Eq. (11), the dimensionless form of Eq. (4) is derived. That is

$\frac{u K_{R}}{h_{1} \bar{u}}=D \frac{\partial^{2} C}{\partial y^{2}}-D_{T} \frac{\partial^{2} T_{\mathrm{f}}}{\partial y^{2}}$.

Since the second derivative of the non-Newtonian, nondimensional fluid temperature is determined from the form of Eq. (20b), only the dimensionless form of boundary conditions of Eq. (6a) and (6b) is required to solve Eq. (29). These are as follows:

$\phi^{\prime \prime}(0)=\frac{S r \cdot k}{Y_{1} \cdot \varepsilon} \theta_{\mathrm{f}}^{\prime}(0)$,

$\phi\left(-Y_{1}\right)=1$.

By applying parts (a) and (b) of Eq. (30), it is possible to determine analytically the second derivative of dimensionless concentration profiles on the cross section, which is

$\phi^{\prime \prime}(Y)=\frac{S r \cdot k}{Y_{1} \cdot \varepsilon} \theta_{\mathrm{f}}^{\prime \prime}(Y)+\frac{\gamma D_{1}\left(\operatorname{Cosh}\left(S Y_{1}\right)-\operatorname{Cosh}(S Y)\right)}{Y_{1}^{2}}$.

Finally, substituting Eq. (28) into Eq. (26), the dimensionless concentration equation, containing two transverse and axial cross sections, can be expressed as follows:

$$
\begin{aligned}
\phi(Y)= & F_{1}+F_{2} Y+F_{3} Y^{2}+F_{4} \operatorname{Cosh}(2 S Y) \\
& +F_{5} \operatorname{Cosh}(S Y)+F_{6} \operatorname{Cosh}(\alpha Y) \\
\phi(X, Y)= & \frac{\gamma X}{P e Y_{1} \xi}+F_{1}+F_{2} Y+F_{3} Y^{2}+F_{4} \operatorname{Cosh}(2 S Y) \\
& +F_{5} \operatorname{Cosh}(S Y)+F_{6} \operatorname{Cosh}(\alpha Y)
\end{aligned}
$$

\section{Nusselt number}

The heat convection coefficient on the upper and lower walls of the channel can be expressed by

$H_{\mathrm{w} 1}=\frac{q_{1}^{\prime \prime}}{T_{\mathrm{w} 1}-\bar{T}_{\mathrm{f}}}$,

$H_{\mathrm{w} 2}=\frac{q_{2}^{\prime \prime}}{T_{\mathrm{w} 2}-\bar{T}_{\mathrm{f}}}$

Considering dimensionless parameters presented in Table 1, the Nusselt number is taken by

$\mathrm{Nu}_{\mathrm{w} 1}=\frac{2 H_{\mathrm{w} 1} h_{1}}{k_{\mathrm{ef}}}=\frac{4(Q-1) Y_{1}}{k \bar{\theta}_{\mathrm{f}}}$,

$\mathrm{Nu}_{\mathrm{w} 2}=\frac{2 H_{\mathrm{w} 2} h_{1}}{k_{\mathrm{ef}}}=\frac{4 Q Y_{1}}{k\left(\theta_{\mathrm{w} 2}-\bar{\theta}_{\mathrm{f}}\right)}$.

The non-Newtonian fluid average temperature is obtained by the dimensionless form of Eq. (16) along the channel. That is 
$\bar{\theta}_{\mathrm{f}}=\frac{D_{1}}{2 Y_{1}} \int_{-Y_{1}}^{Y_{1}} \theta_{\mathrm{f}}\left[\operatorname{Cosh}\left(S Y_{1}\right)-\operatorname{Cos}(S Y)\right] \mathrm{d} Y$.

\section{Entropy generation}

The entropy generation in the current system raises from various irreversible sources. The contribution of heat and mass transfer and friction between the fluid and solid surface to exergy destruction cause lower energy quality and weaker hydrothermal performance of the current system.

The potential sources of entropy generation in the typical microchannel are presented as the following:

$\dot{S}_{\mathrm{w} 2}^{\prime \prime \prime}=\frac{k_{2}}{T_{2}^{2}}\left[\left(\frac{\partial T_{2}}{\partial x}\right)^{2}+\left(\frac{\partial T_{2}}{\partial y}\right)^{2}\right]$,
$\dot{S}_{\mathrm{S}}^{\prime \prime \prime}=\frac{k_{\mathrm{es}}}{T_{\mathrm{S}}^{2}}\left[\left(\frac{\partial T_{\mathrm{s}}}{\partial x}\right)^{2}+\left(\frac{\partial T_{\mathrm{s}}}{\partial y}\right)^{2}\right]-\frac{h_{\mathrm{sf}} a_{\mathrm{sf}}\left(T_{\mathrm{S}}-T_{\mathrm{f}}\right)}{T_{\mathrm{S}}}$,

$\dot{S}_{\mathrm{f}}^{\prime \prime \prime}=\frac{k_{\mathrm{ef}}}{T_{\mathrm{f}}^{2}}\left[\left(\frac{\partial T_{\mathrm{f}}}{\partial x}\right)^{2}+\left(\frac{\partial T_{\mathrm{f}}}{\partial y}\right)^{2}\right]+\frac{h_{\mathrm{sf}} a_{\mathrm{sf}}\left(T_{\mathrm{S}}-T_{\mathrm{f}}\right)}{T_{\mathrm{f}}}$,

$\dot{S}_{\mathrm{FF}}^{\prime \prime \prime}=\frac{\mu_{\mathrm{f}}}{\kappa T_{\mathrm{f}}} u^{2}+\frac{\mu_{\mathrm{eff}}}{T_{\mathrm{f}}}\left(\frac{\mathrm{d} u}{\mathrm{~d} y}\right)^{2}$,

$$
\begin{aligned}
\dot{S}_{\mathrm{DI}}^{\prime \prime \prime}= & \frac{R D}{C}\left[\left(\frac{\partial C}{\partial x}\right)^{2}+\left(\frac{\partial C}{\partial y}\right)^{2}\right] \\
& +\frac{R D}{T_{\mathrm{f}}}\left[\left(\frac{\partial C}{\partial x}\right)\left(\frac{\partial T_{\mathrm{f}}}{\partial x}\right)+\left(\frac{\partial C}{\partial y}\right)\left(\frac{\partial T_{\mathrm{f}}}{\partial y}\right)\right],
\end{aligned}
$$

$\dot{S}_{\mathrm{w} 1}^{\prime \prime \prime}=\frac{k_{1}}{T_{1}^{2}}\left[\left(\frac{\partial T_{1}}{\partial x}\right)^{2}+\left(\frac{\partial T_{1}}{\partial y}\right)^{2}\right]$.

The dimensionless results of Eq. (37a-37f) are

$$
\begin{aligned}
& N_{\mathrm{i}}=\frac{\dot{S}_{i}^{\prime \prime \prime} h_{2}^{2}}{k_{\mathrm{es}}}, \quad i=w 1, s, f, D I, w 2 \\
& \omega=\frac{\left(q_{1}^{\prime \prime}+q_{2}^{\prime \prime}\right) h_{2}}{2 k_{\mathrm{es}} T_{\mathrm{w}, \mathrm{in}}}, \\
& \varphi=\frac{R D C_{0}}{k_{\mathrm{es}}}
\end{aligned}
$$

in which $\dot{S}_{\mathrm{w} 1}^{\prime \prime \prime}$ and $\dot{S}_{\mathrm{w} 2}^{\prime \prime \prime}$ are the volumetric entropy generation rate from the lower and upper walls. Further, $\dot{S}_{\mathrm{s}}^{\prime \prime \prime}, \dot{S}_{\mathrm{f}}^{\prime \prime \prime}$ and $\dot{S}_{\mathrm{DI}}^{\prime \prime \prime}$ are the volumetric entropy generation in the porous solid, fluid and due to mass transfer, respectively. Further, $R$ denotes the specific gas constant, $N$ refers to the dimensionless irreversibility, $\omega$ is the dimensionless heat flux and $\varphi$ is the irreversibility distribution ratio.

In order to provide a simple substrate for investigating the entropy generation and evaluating its distribution for different irreversible sources, the equations for the solid and fluid phases in the porous medium are described as follows:

$N_{\mathrm{w} 1}=\frac{k_{e 1} \omega^{2}}{\left(\omega \theta_{1}+1\right)^{2}}\left[\left(\frac{\partial \theta_{1}}{\partial X}\right)^{2}+\left(\frac{\partial \theta_{1}}{\partial Y}\right)^{2}\right],-1 \leq Y<-Y_{1}$

$$
\begin{aligned}
N_{\mathrm{s}}= & \frac{\omega^{2}}{\left(\omega \theta_{\mathrm{s}}+1\right)^{2}}\left[\xi^{2}\left(\frac{\partial \theta_{\mathrm{s}}}{\partial X}\right)^{2}+\left(\frac{\partial \theta_{\mathrm{s}}}{\partial Y}\right)^{2}\right] \\
& -\frac{B i \cdot \omega\left(\theta_{\mathrm{s}}-\theta_{\mathrm{f}}\right)}{\left(\omega \theta_{\mathrm{s}}+1\right)},-Y_{1} \leq Y<-Y_{1}
\end{aligned}
$$

$$
\begin{aligned}
N_{\mathrm{f}}= & \frac{k \omega^{2}}{\left(\omega \theta_{\mathrm{f}}+1\right)^{2}}\left[\xi^{2}\left(\frac{\partial \theta_{\mathrm{f}}}{\partial X}\right)^{2}+\left(\frac{\partial \theta_{\mathrm{f}}}{\partial Y}\right)^{2}\right] \\
& +\frac{\mathrm{Bi} \cdot \omega\left(\theta_{\mathrm{s}}-\theta_{\mathrm{f}}\right)}{\left(\omega \theta_{\mathrm{f}}+1\right)},-Y_{1} \leq Y<-Y_{1}
\end{aligned}
$$

$$
\begin{aligned}
N_{\text {DI }}= & \frac{\varphi}{\phi}\left[\xi^{2}\left(\frac{\partial \phi}{\partial X}\right)^{2}+\left(\frac{\partial \phi}{\partial Y}\right)^{2}\right] \\
& +\frac{\varphi . \omega}{\left(\omega \theta_{\mathrm{f}}+1\right)}\left[\xi^{2}\left(\frac{\partial \phi}{\partial X}\right)\left(\frac{\partial \theta_{\mathrm{f}}}{\partial X}\right)+\left(\frac{\partial \phi}{\partial Y}\right)\left(\frac{\partial \theta_{\mathrm{f}}}{\partial Y}\right)\right], \\
& -Y_{1} \leq Y<-Y_{1}
\end{aligned}
$$

$N_{w 2}=\frac{k_{e 2} \omega^{2}}{\left(\omega \theta_{2}+1\right)^{2}}\left[\left(\frac{\partial \theta_{2}}{\partial X}\right)^{2}+\left(\frac{\partial \theta_{2}}{\partial Y}\right)^{2}\right] . Y_{1} \leq Y<1$

By adding Eqs. (41b) and (42c), the volumetric entropy generation expressions are defined as follows:

$N_{\mathrm{f}, \mathrm{ht}}=\frac{\omega^{2}}{\left(\omega \theta_{\mathrm{s}}+1\right)^{2}}\left[\xi^{2}\left(\frac{\partial \theta_{\mathrm{s}}}{\partial X}\right)^{2}+\left(\frac{\partial \theta_{\mathrm{s}}}{\partial Y}\right)^{2}\right]$,

$N_{\mathrm{f}, \mathrm{ht}}=\frac{k \omega^{2}}{\left(\omega \theta_{\mathrm{f}}+1\right)^{2}}\left[\xi^{2}\left(\frac{\partial \theta_{\mathrm{f}}}{\partial X}\right)^{2}+\left(\frac{\partial \theta_{\mathrm{f}}}{\partial Y}\right)^{2}\right]$. 
The volumetric entropy generation in the porous media is equal to the sum of the equations applicable to the porous media.

$N_{\text {int }}=\frac{\text { Bi. } \omega^{2}\left(\theta_{\mathrm{f}}-\theta_{\mathrm{s}}\right)^{2}}{\left(\omega \theta_{\mathrm{s}}+1\right)\left(\omega \theta_{\mathrm{f}}+1\right)}$.

The total entropy generation defines the entropy produced by the heat transfer, viscous dissipative and concentration gradient for each point in the $X$ and $Y$ directions. That is

$N_{\mathrm{pm}}=N_{\mathrm{s}, \mathrm{ht}}+N_{\mathrm{f}, \mathrm{ht}}+N_{\mathrm{int}}+N_{\mathrm{DI}}$.

The total entropy generation on the whole microchannel is obtained by sweeping two-segmental integration on the physical domain, which equals

$N_{\text {Tot }}=\int_{-1}^{1} \int_{0}^{1} N_{\mathrm{pm}} \mathrm{d} X \mathrm{~d} Y$.

Table 2 Default values of the dimensionless parameters

\begin{tabular}{llll}
\hline $\begin{array}{l}\text { Dimensionless } \\
\text { parameter }\end{array}$ & Default value & $\begin{array}{l}\text { Dimensionless } \\
\text { parameter }\end{array}$ & Default value \\
\hline $\mathrm{Bi}$ & 1.0 & $k$ & 0.05 \\
$\mathrm{Da}$ & 0.1 & $k_{\mathrm{e} 1}$ & 0.5 \\
$n$ & 1.3 & $k_{\mathrm{e} 2}$ & 0.5 \\
$\mathrm{Pe}$ & 10 & $\varepsilon$ & 0.95 \\
$\mathrm{Pr}$ & 5.0 & $\gamma$ & 0.95 \\
$\mathrm{Re}$ & 150 & $\omega$ & 0.001 \\
$\mathrm{Sr}$ & 0.7 & $\varphi$ & 0.01 \\
$Q$ & 0.75 & $X_{0}$ & 0.1 \\
$Y_{1}$ & 0.8 & $\xi$ & 0.05 \\
\hline
\end{tabular}

\section{Numerical simulation and validation}

The equations derived analytically in Section "Theoretical and numerical methods" provide the general solution of the dimensionless governing equations. Although it is possible to derive fully analytical solutions for the current problem, the non-Newtonian nature of the considered fluid makes such solution immensely complicated and therefore of little use. Hence, to evaluate the unknown coefficients and find the particular solutions, a numerical approach was taken. The details of numerical methodology along with the specification of the system configuration are given in this section.

\section{Description of the system configuration}

Figure 1 shows a schematic of the geometry considered in the current study. The microchannel is fully filled with porous materials, with two thick walls, subjected to constant but non-equal heat fluxes with a catalytic layer on the inner microchannel surface. A non-Newtonian, power-law fluid flow enters the left side of the microchannel and, after passing through the porous media, leaves from the right side of the system.

The problem outlined in Section "Theoretical and numerical methods" was solved using various non-dimensional and dimensional parameters that are presented for quantitative analysis by the default values in Tables 2 and 3 , respectively. It is noted that the values of parameters in Table 2 were chosen in a way that the results become comparable with the existing data in the literature for Newtonian fluids $[1,3]$.

\section{Grid independency and validation}

The non-dimensionalized governing equations derived in Section "Theoretical and numerical methods" (Eqs. (7), (17a), (17b), (18a) and (18b) and (31) along with boundary
Table 3 Default values of the dimensional parameters

\begin{tabular}{|c|c|c|c|}
\hline Dimensional parameter & Default value & Dimensional parameter & Default value \\
\hline$h_{1}$ & $400 \mu \mathrm{m}$ & $k_{2}$ & $53.661 \mathrm{~W} \mathrm{~K}^{-1} \mathrm{~m}^{-2}$ \\
\hline$h_{2}$ & $400 \mu \mathrm{m}$ & $k_{\mathrm{es}}$ & $40 \mathrm{~W} \mathrm{~K}^{-1} \mathrm{~m}^{-2}$ \\
\hline$L$ & $0.1 \mathrm{~m}$ & $k_{\text {ef }}$ & $0.604675 \mathrm{~W} \mathrm{~K}^{-1} \mathrm{~m}^{-2}$ \\
\hline$\kappa$ & $6.25 \times 10^{-5} \mathrm{~m}^{2}$ & $k_{\mathrm{s}}$ & $53.661 \mathrm{~W} \mathrm{~K}^{-1} \mathrm{~m}^{-2}$ \\
\hline$q_{1}^{\prime \prime}$ & $150 \mathrm{~W} \mathrm{~m}^{-2}$ & $D$ & $2.91 \times 10^{-4} \mathrm{~m}^{2} \mathrm{~s}^{-1}$ \\
\hline$q_{2}^{\prime \prime}$ & $50 \mathrm{~W} \mathrm{~m}^{-2}$ & $\bar{u}$ & $0.1455 \mathrm{~m} \mathrm{~s}^{-1}$ \\
\hline$C_{0}$ & $5 \mathrm{~kg} \mathrm{~m}^{-3}$ & $D_{\mathrm{T}}$ & $0.16212\left(\mathrm{~m}\left(\mathrm{~K} \mathrm{~kg} \mathrm{~s}^{-1}\right)\right.$ \\
\hline$T_{\mathrm{w}, \text { in }}$ & $314.231 \mathrm{~K}$ & $\mu_{\mathrm{f}}$ & $8.94 \times 10^{-4}\left(\mathrm{~N} \mathrm{~s} \mathrm{~m}^{-2}\right)$ \\
\hline$k_{1}$ & $53.661 \mathrm{~W} \mathrm{~K}^{-1} \mathrm{~m}^{-2}$ & $C_{\mathrm{p}}$ & $4179\left(\mathrm{~J} \mathrm{~kg}^{-1} \mathrm{~K}^{-1}\right)$ \\
\hline
\end{tabular}




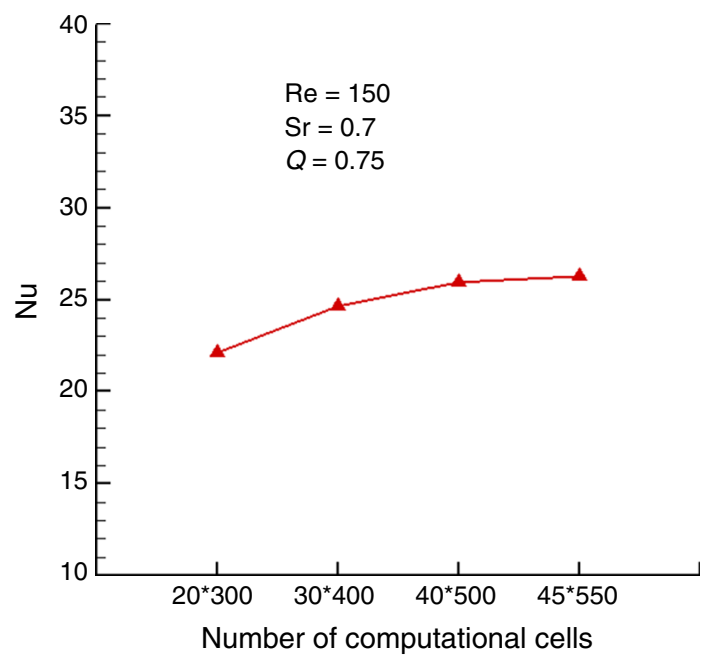

Fig. 2 Grid independency test

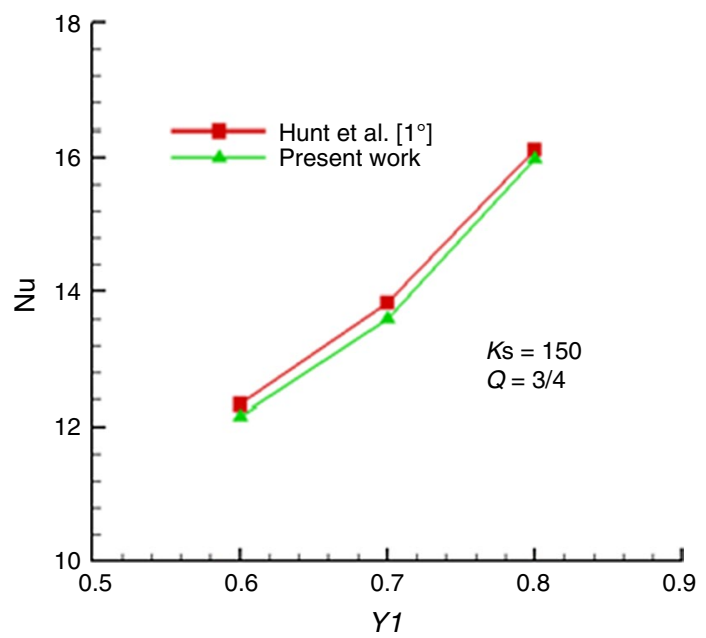

Fig. 3 Comparison of Nusselt number between the present work and the results of Hunt et al. [1] in three different values of wall thickness, $\left(Y_{1}\right)$

conditions of Eq. (19)) were solved numerically through using a finite volume technique. Four mesh densities including $20 \times 300,30 \times 400,40 \times 500$ and $45 \times 550$ grids were tested. It was observed that the mesh sizes of $40 \times 500$ and $45 \times 550$ are perfectly consistent and have a relative error of $1.35 \%$ for Nusselt number. Thus, in the current study a mesh containing $40 \times 500$ cells was used (Fig. 2).

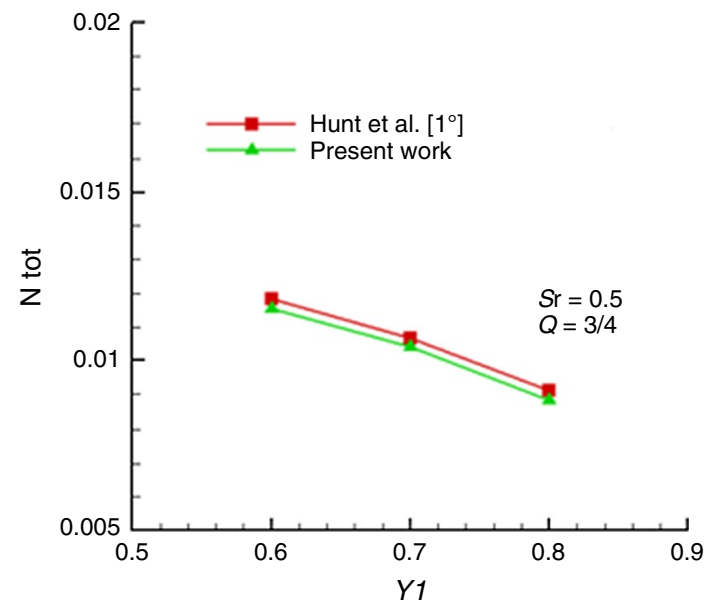

Fig. 4 Comparison of entropy generation between the present work and the results of Hunt et al. [1] in three different values of wall thickness, $\left(Y_{1}\right)$

The present numerical data are compared with the closed-form analytical results of Hunt et al. [1] for Nusselt number and entropy generation (Figs. 3 and 4) in the limit case that power-law fluid approaches a Newtonian fluid at different values of wall thickness $\left(Y_{1}\right)$. Evidently, the two data sets exhibit very good agreement and thus the analytical solutions are deemed to be valid.

\section{Results and discussion}

In this section, an emphasis is put on the influences of nonNewtonian fluid characteristics upon the thermal behavior of the micro-system. In the interest of brevity, other effects are not discussed.

\section{Detailed comparison of transport phenomena and entropy generation between Newtonian and power-law fluids}

Figure 5 shows the dimensionless temperature contours drawn for the solid and fluid phases of the porous medium for different values of power index. It should be noted that the dimensionless temperature in this figure can take negative or positive values. The temperature contours in Fig. 5 indicate that with increasing power index, as the fluid tends to thicken and increase the viscosity and thus increase the shear stress, the heat transfer rate decreases. This can be 
Fig. 5 Contours of dimensionless temperature for varying values of the power-law index $(n)$ at $\left(Y_{1}=0.7\right)$, a solid phase $n=0.5$, $\mathbf{b}$ fluid phase $n=0.5$, c solid phase $n=1.0, \mathbf{d}$ fluid phase $n=1.0$, e solid phase $n=1.5, \mathbf{f}$ fluid phase $n=1.5$ (a)

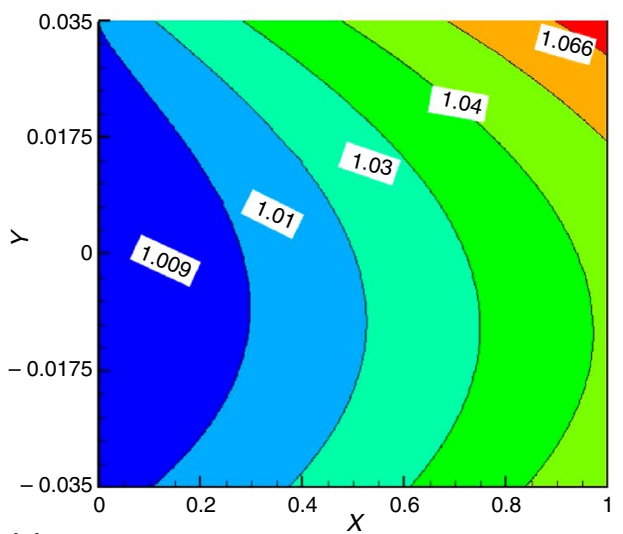

(c)

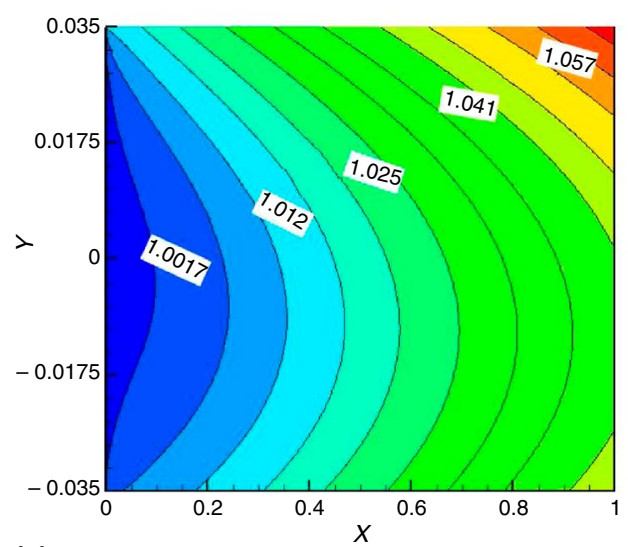

(e)

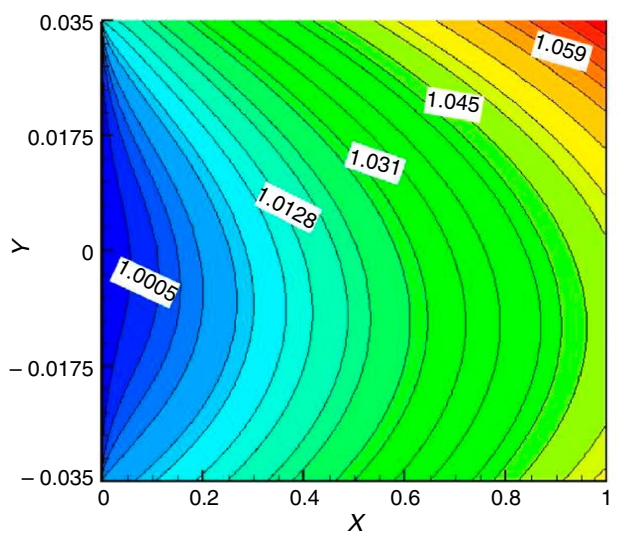

(b)

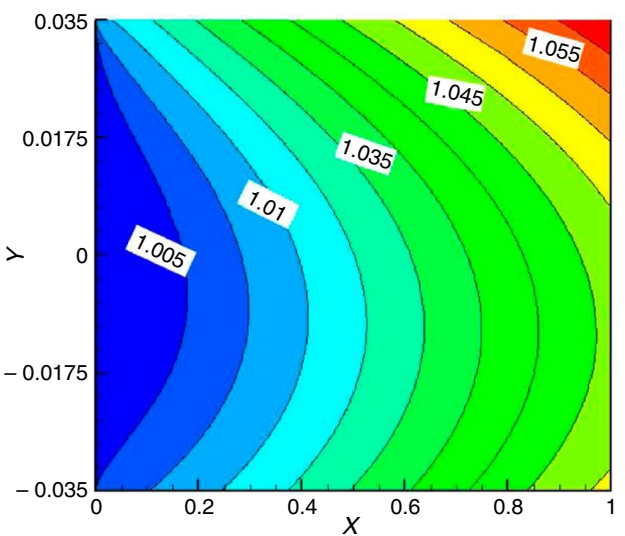

(d)

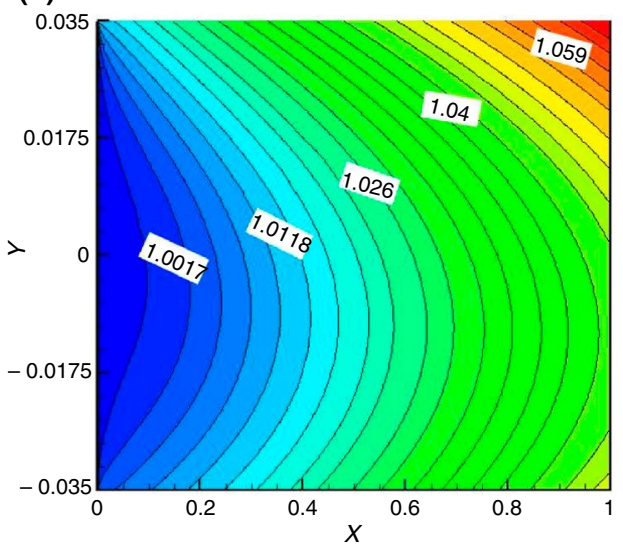

(f)

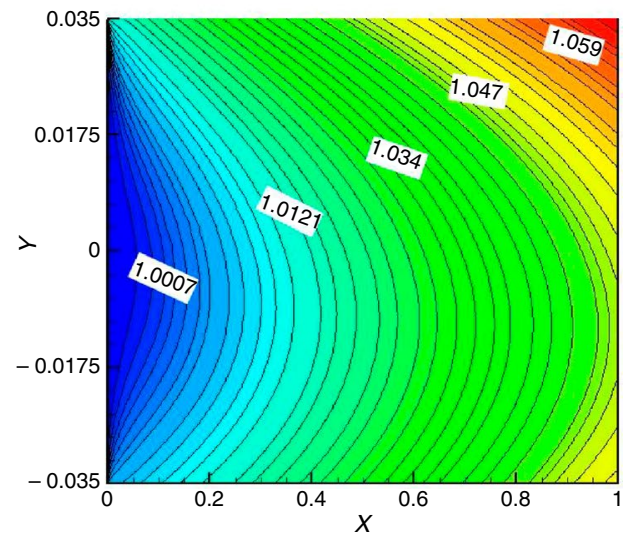


Fig. 6 Contours of local entropy generation for the fluid phase for varying Damköhler number, $\gamma, \mathbf{a} n=1.0, \gamma=0.8$, b $n=1.3, \gamma=0.8, \mathbf{c}$ $n=1.0, \gamma=1.2, \mathbf{d}$ $n=1.3, \gamma=1.2$.

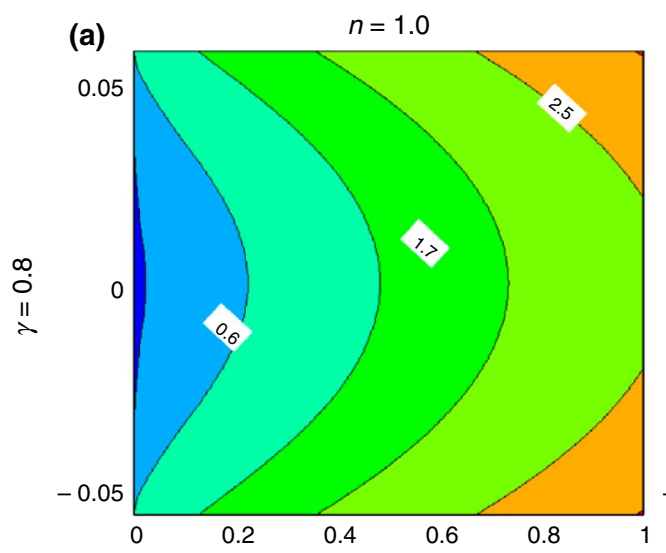

(b) $\quad n=1.3$

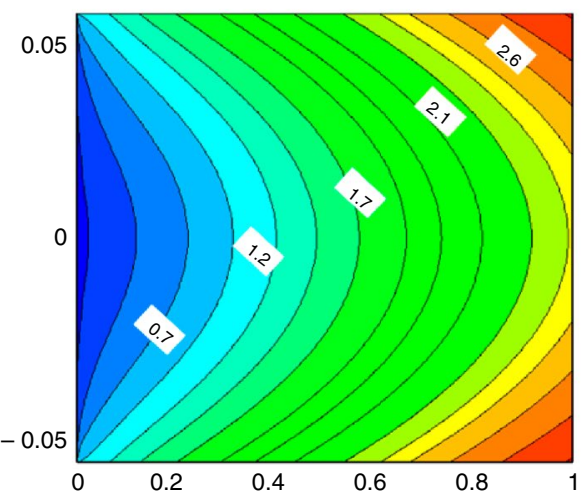

(c)

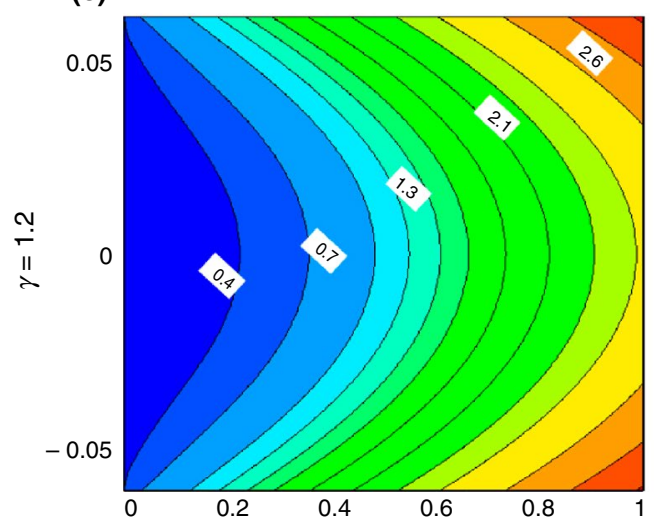

deduced from the fact that the fluid temperature takes larger values of dimensionless temperature at higher values of power index. Since the dimensionless temperature expresses the difference between the temperature of fluid and that of the wall, higher values of dimensionless temperature imply larger difference between fluid and wall temperature. For the current iso-flux problem, this means weakened convective heat transfer. This argument will be later supported by calculation of Nusselt number.

Figures 6 and 7 show the contours related to the local entropy generation inside the fluid phase of the microchannel. It is important to note that entropy generation is only calculated for the fluid phase and porous media and the solid wall are excluded from the analyses of local entropy generation. Also, as depicted by Eq. (41d) the mass transfer irreversibilities are included in the current analysis.
Figure 6 shows the total entropy variations for the two power-law indices with two different Damköhler numbers for the fluid phase. It is clarified that the value of Damköhler number influences the rate of mass transfer and thus can affect the irreversibility. As can be seen in this figure, for a value of Damköhler number less than one, an increment in the value of the power-law index intensifies the generation of entropy. This is due to the enhancement of friction irreversibilities at higher values of power-law index. More importantly, as shown earlier, the rate of heat transfer decreases at higher values of power-law index, which in turn strengthens the temperature gradients and thus increases the generation of entropy. Interestingly, this behavior is inversed at higher value of Damköhler number. This is because of 
Fig. 7 Contours of local entropy generation for the fluid phase for varying Soret number, $S r$, a $S r=0.5, n=1.0$, b $S r=0.7, n=1.3$, c $S r=0.5, n=1.0, \mathbf{d}$ $S r=0.7, n=1.3$
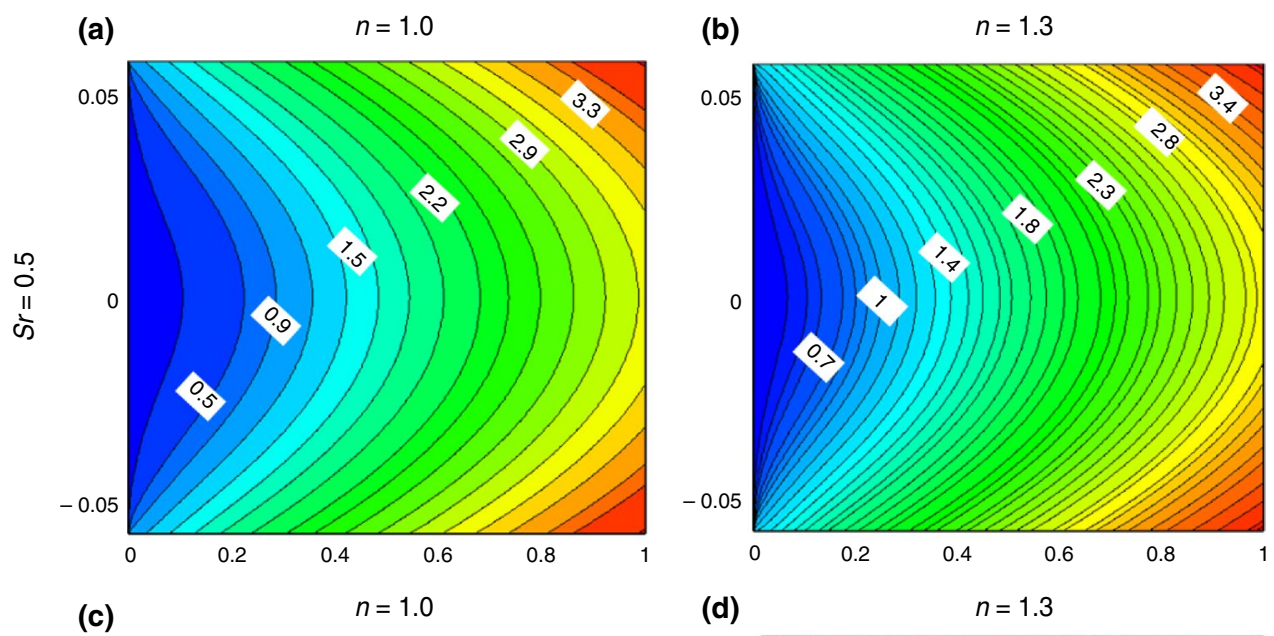

(d)

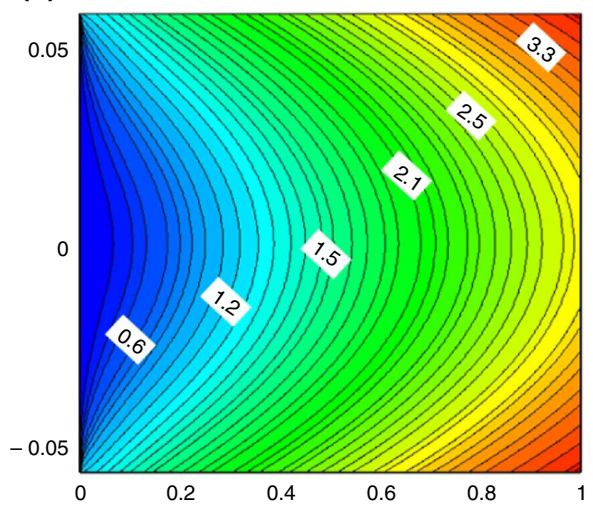

the dominance of mass transfer in entropy generation in the current problem $[1,35,36]$, which can overrule other effects.

Figure 7 illustrates the influences of changes in Soret number upon the local entropy generation in the fluid phase. This has been done for two different values of the power-law index corresponding to Newtonian and non-Newtonian fluids. Figure 7 indicates that for a constant power-law index, in either of Newtonian and non-Newtonian fluids, Soret number growth causes a reduction in the local irreversibility. This can be explained by noting that in the current problem irreversibility is dominated by that of mass transfer [34-36]. Magnifying the value of Soret number enhances the process of mass transfer and hence reduces the irreversibility associated with the transport of mass. Thus, the local entropy generation drops. Further, Fig. 7 shows that increasing the value of power-law index at a fixed Soret number leads to a slight intensification of the local entropy generation. As already discussed, this is mostly due to the magnification of thermal irreversibilities following the fluid temperature gradient strengthening.

According to Fig. 8, increasing the power-law index results in the reduction in Nusselt number. This reduction can be quite significant such that by increasing the power-law index from 0.4 to 1.8 , a drop of around $50 \%$ is observed in Nusselt number for all the investigated cases. This is an important result and shows that deviation from the state of Newtonian fluid can majorly affect the rate of heat transfer. Hence, close attention should be paid to 


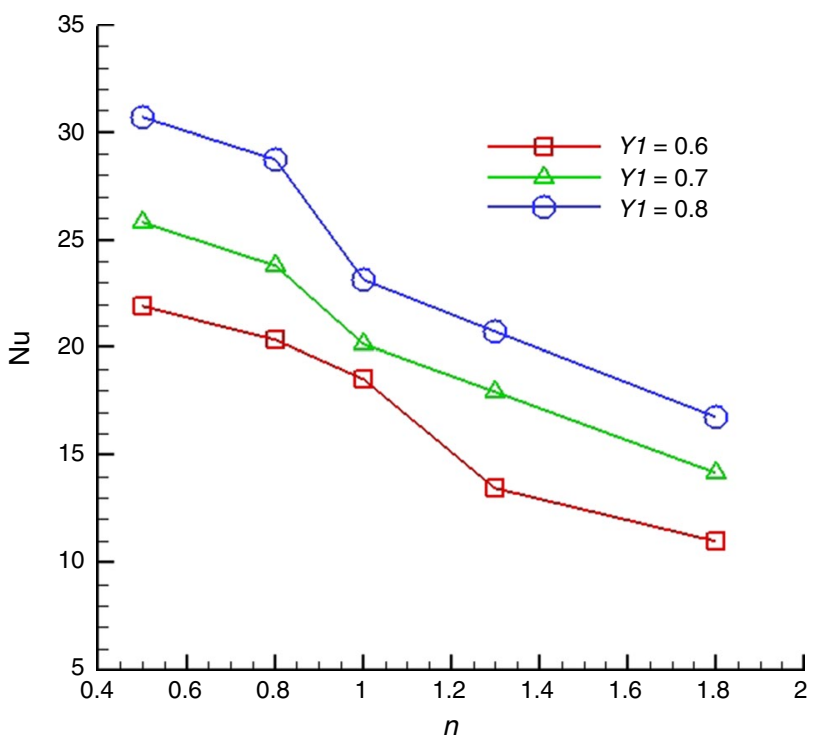

Fig. 8 Nusselt Number against the power-law index $(n)$ for different values of wall thickness, $\left(Y_{1}\right)$

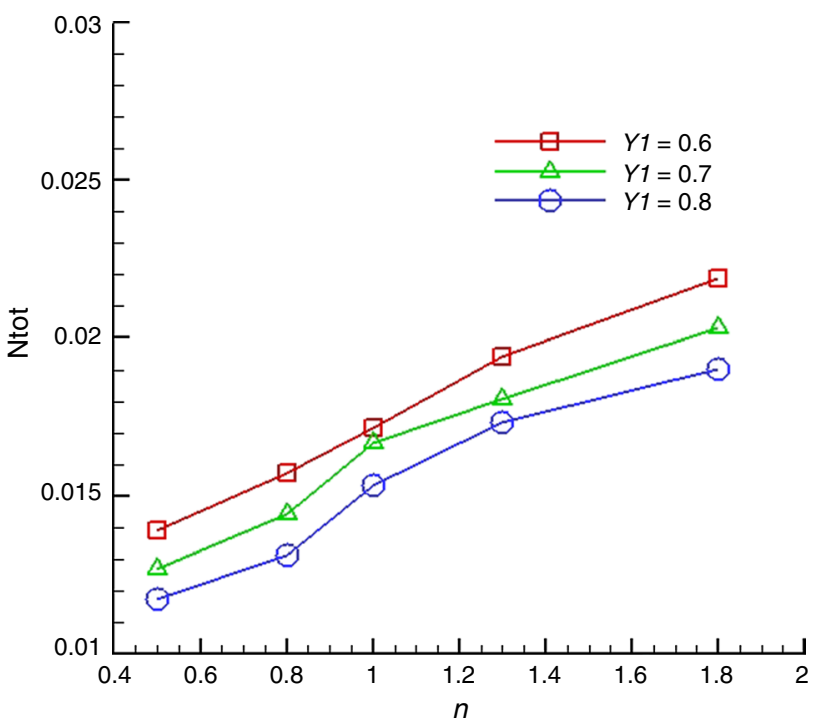

Fig. 9 Total entropy generation against the values of power-law index $(n)$ for different values of wall thickness, $\left(Y_{1}\right)$

possible non-Newtonian behaviors of the working fluids in microchannels and microreactors. Figure 8 further shows the pronounced effects of the wall thickness upon Nusselt number. In keeping with the results reported in the literature
$[33,34]$, it is shown that thicker walls act as thermal resistance and thus tend to reduce the Nusselt number. This figure clearly shows that alteration of wall thickness by around $30 \%$ can change the value of Nusselt number by more than $40 \%$. Thus, wall thickness is an essential parameter dominating the rate of heat transfer in microchannels.

Figure 9 illustrates the effects of power-law index on the total generation of entropy. Clearly, variations in the power-law index strongly affect the total entropy generation. According to this figure, an increase in the power-law index from 0.4 to 1.8 can boost the total entropy by more than $50 \%$. As already discussed, decreasing $Y_{1}$ (thickening the walls) also contributes to this increase. Once again, impediment of heat convection process by increases in the value of power-law index (as already discussed) is responsible for this trend.

\section{Parametric studies on the effects of physical properties of power-law fluids}

In Fig. 10, temperature contours for non-Newtonian fluids for two different values of $Y_{1}$ are depicted. The righthand side contours correspond to the solid phase, and the left-hand side contours correspond to the fluid phase. It is observed that by increasing $Y_{1}$, that is by making the wall thinner, the deflection of temperature contours increases. Thus, at any cross section in the microchannel, the transversal temperature variation increases. As shown previously $[1,34,35]$ and also further supported in the later sections, this causes an increase in the overall heat transfer rate of the system.

Figure 11 shows the distribution of dimensionless fluid temperature inside the porous microchannel for different values of Reynolds number and the power-law index. This figure essentially compares the case of Newtonian fluid to that of non-Newtonian fluid. Evidently, regardless of the value of Reynolds number, the axial temperature increase along the microchannel for the non-Newtonian fluid is larger than that of the Newtonian fluid. For the current constant heat flux problem, this implies smaller convection coefficient for the non-Newtonian fluid flow. This finding will be later verified by calculating the Nusselt number for the two types of fluid. Further, the increase in Nusselt number has resulted in a general drop of the dimensionless temperature at any given point inside the microchannel. This is to be expected, as in general, increases in Reynolds number (on the basis of the microchannel height) lead to 
Fig. 10 Contours of dimensionless temperature at $n=0.5$ for varying values of the wall thickness, $Y_{1}$, a solid phase $Y_{1}=0.6$, b fluid, $Y_{1}=0.6$, c solid phase, $Y 1=0.9$, d fluid phase, $Y_{1}=0.9$ (a)

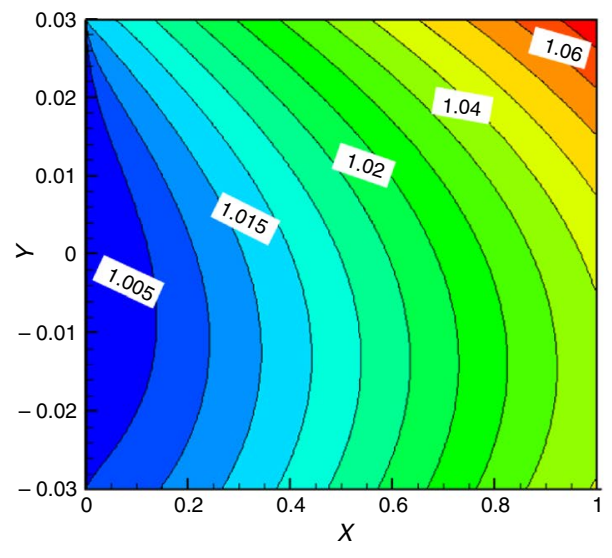

(c)

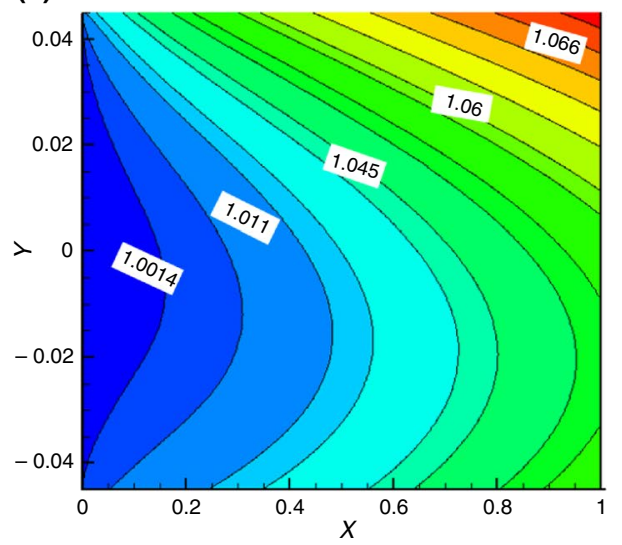

(b)

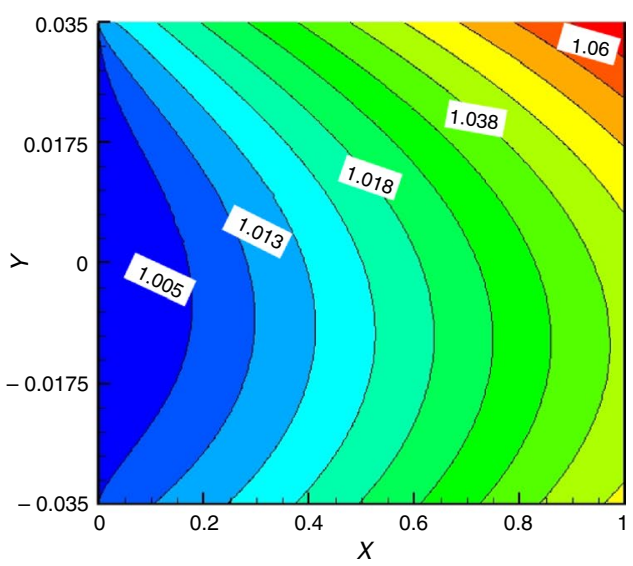

(d)

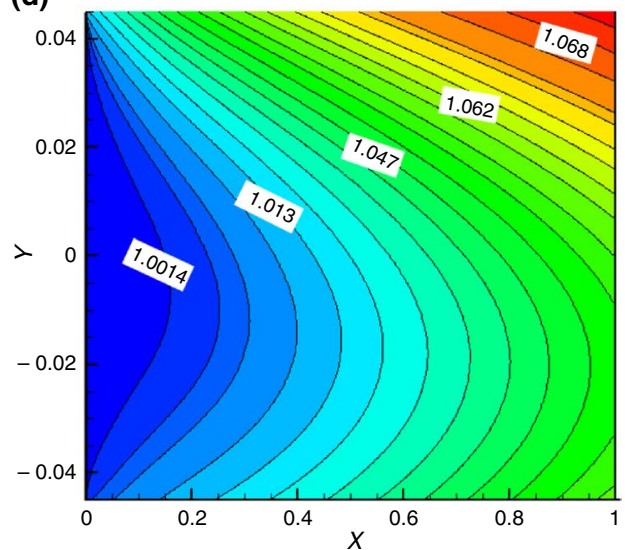

improved heat convection coefficient and thus result in lower temperatures.

Figure 12a shows the effect of variation in the wall heat flux on the Nusselt number. As can be seen by increasing the value of wall heat flux ratio, Q, performed by strengthening the thermal asymmetry of the problem, the Nusselt number increases. This increment in Nusselt number is a consequence of changes in the shape of temperature contours and resultant modification of temperature gradient on the surface of the wall [33-36]. The effects of Darcy number on the Nusselt number are demonstrated in Fig. 12b.

As can be seen, the smaller the Darcy number, meaning the lower the permeability, the rate of heat transfer is increased. This is a well-known behavior of the Nusselt number $[1,5,11,15]$ and shows the consistency of the current Non-Newtonian simulations with those conducted earlier on Newtonian fluids. In Fig. 12c, the effect of variations in the conductivity coefficient of the solid part of the porous media $\left(k_{\mathrm{s}}\right)$ is presented. This graph shows that with augmentation of $k_{\mathrm{s}}$ the value of Nusselt number increases. This can be attributed to the increase in heat transfer between the fluid and the solid part of the porous medium, which is enhanced by boosting the conductivity of the porous solid [37, 38].

Figure 13 shows the influences of power-law index upon the values of Nusselt number and total entropy generation against the Reynolds number. As a general trend, both Nusselt 
Fig. 11 Contours of dimensionless temperature for the fluid phase for varying Soret number, $S r, \mathbf{a} \operatorname{Re}=50, n=1.0$, b $\operatorname{Re}=50, n=1.3$, $\mathbf{R e}$ $=100, n=1.0, \mathbf{d} \operatorname{Re}=100$, $n=1.3, \mathbf{e} \operatorname{Re}=150, n=1.0 \mathbf{f}$ $\operatorname{Re}=160, n=1.3$

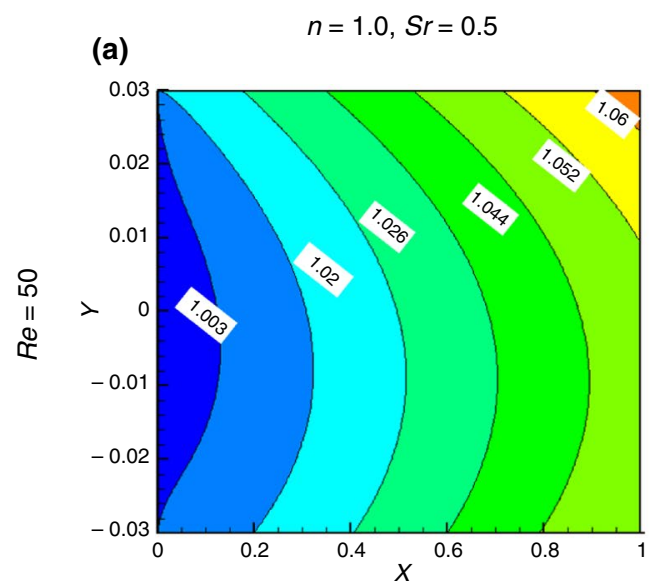

(c)

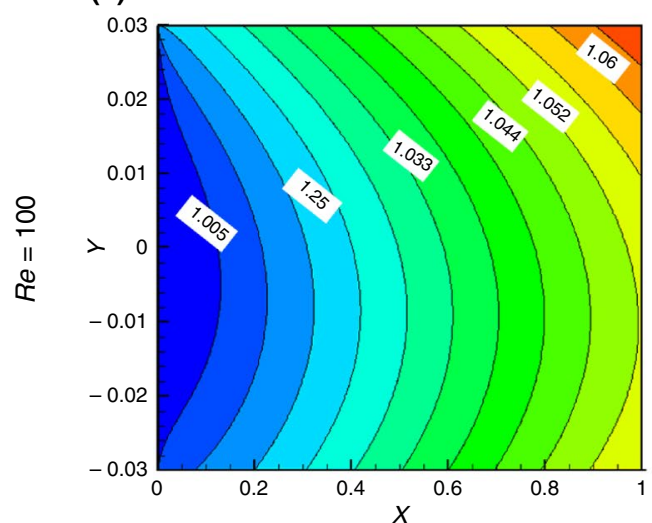

(e)

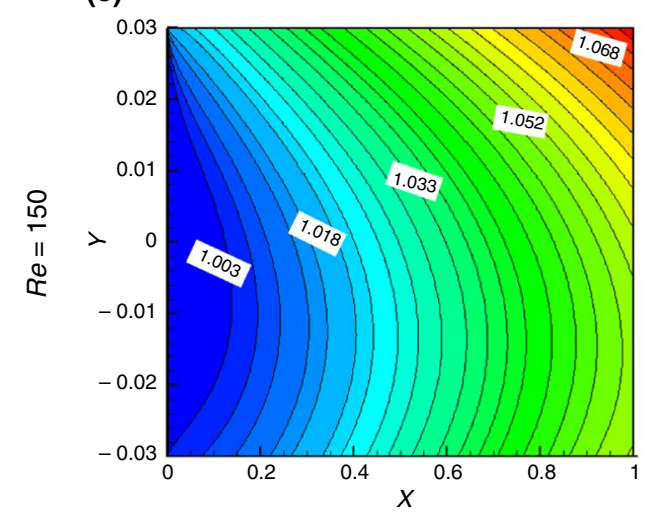

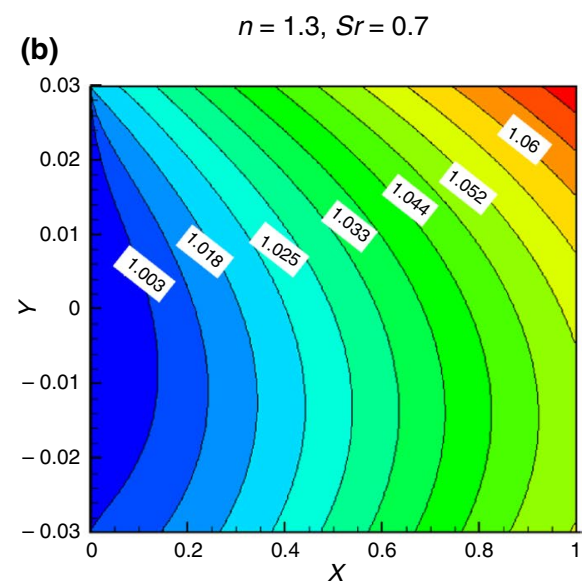

(d)

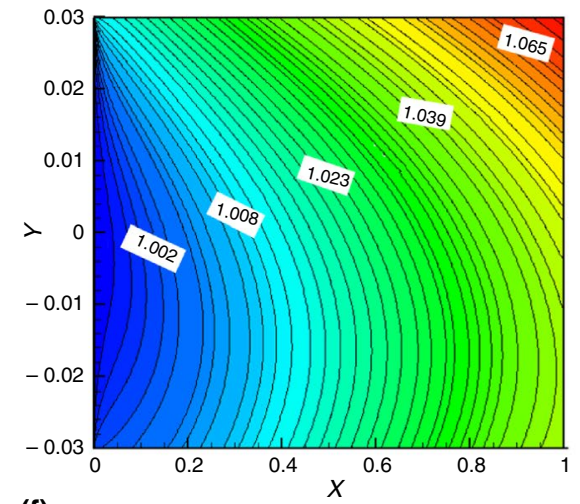

(f)

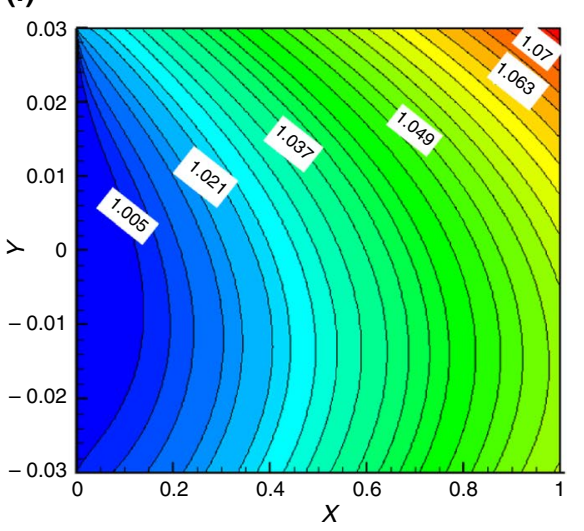

number and total entropy generation increase monotonically with Reynolds number. It is to be expected that Nusselt number in forced convection is often strongly correlated with flow Reynolds number. However, the growth of total entropy generation with Reynolds number may sound counterintuitive. This is because Nusselt number rising relaxes the internal temperature gradients inside the microchannel, and this results in the thermal irreversibility reduction. These apparently 
(a)

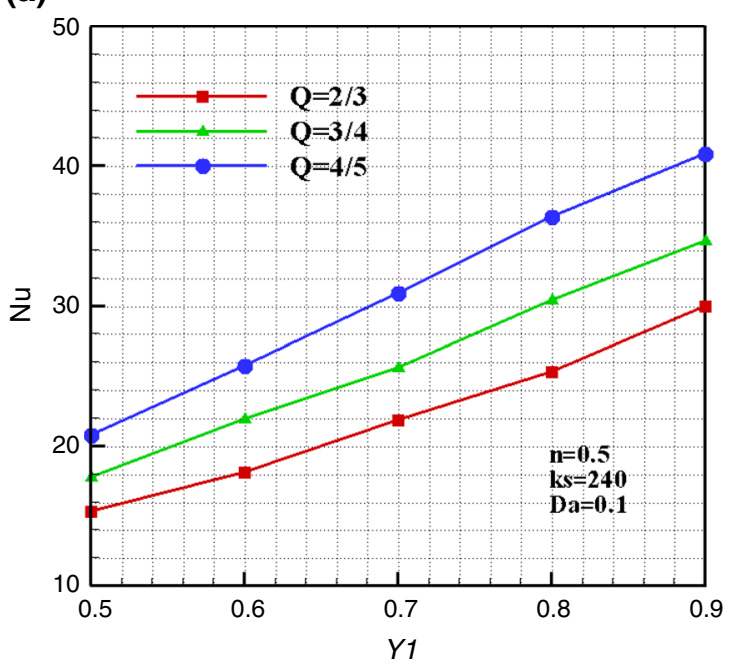

(b)

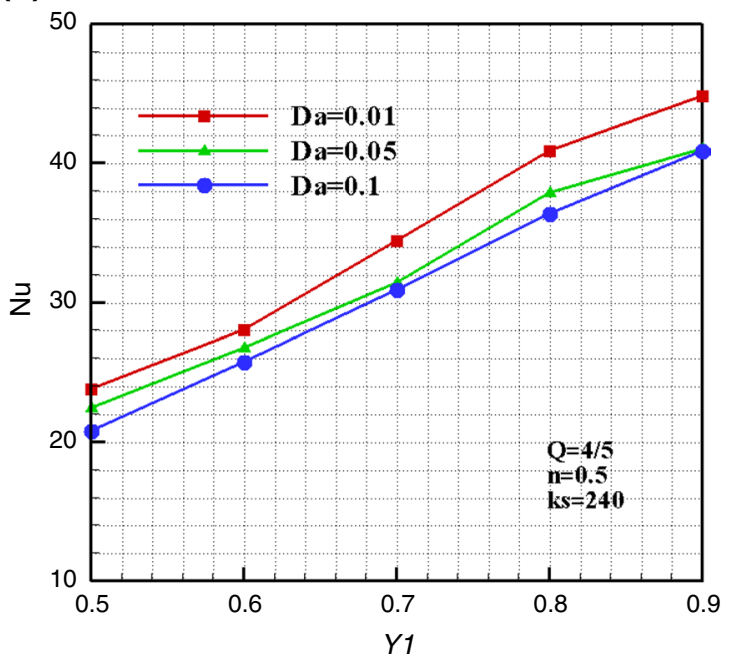

(c)

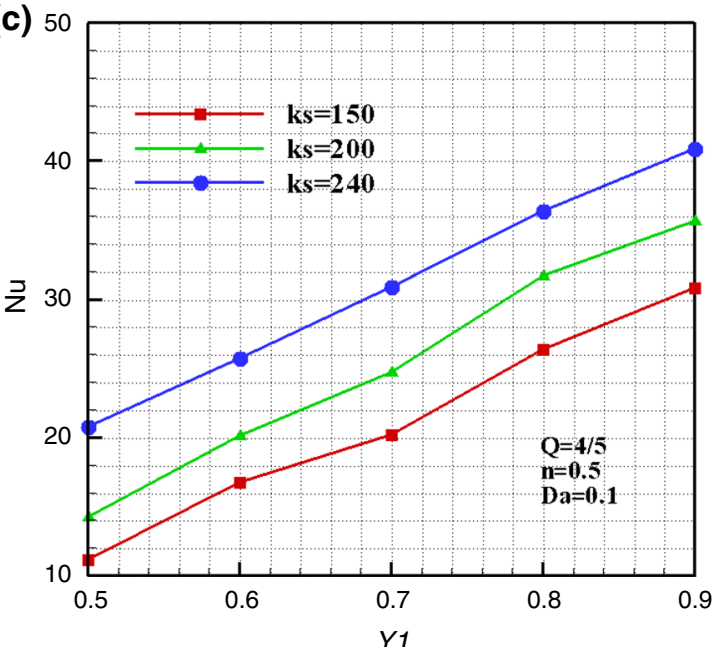

Fig. 12 Nusselt number versus the lower wall thickness, $Y_{1}$, a on the upper wall for different values of $Q$, $\mathbf{b}$ on the lower wall for different values of Darcy number, $\mathbf{c}$ on the upper wall for different values of thermal conductivity of the porous medium, $k_{\mathrm{s}}\left(\mathrm{W} \mathrm{m}^{-1} \mathrm{~K}^{-1}\right)$

contradicting observations can be justified by noting that the entropy generation in the current problem is dominated by flow friction and not heat transfer. Switching to non-Newtonian fluid intensifies the flow friction and thus magnifies the total generation of entropy [39]. Evidently, for all Reynolds numbers, the application of non-Newtonian fluid $(n=1.3)$ leads to a smaller value of Nusselt number (see Fig. 13a). The reduction in heat transfer and the resultant increase in the internal temperature gradient, as well as increases in the frictional losses of the flow due to non-Newtonian effects, are the reasons for augmentation of entropy generation in comparison with that of Newtonian fluid [40, 41]. 

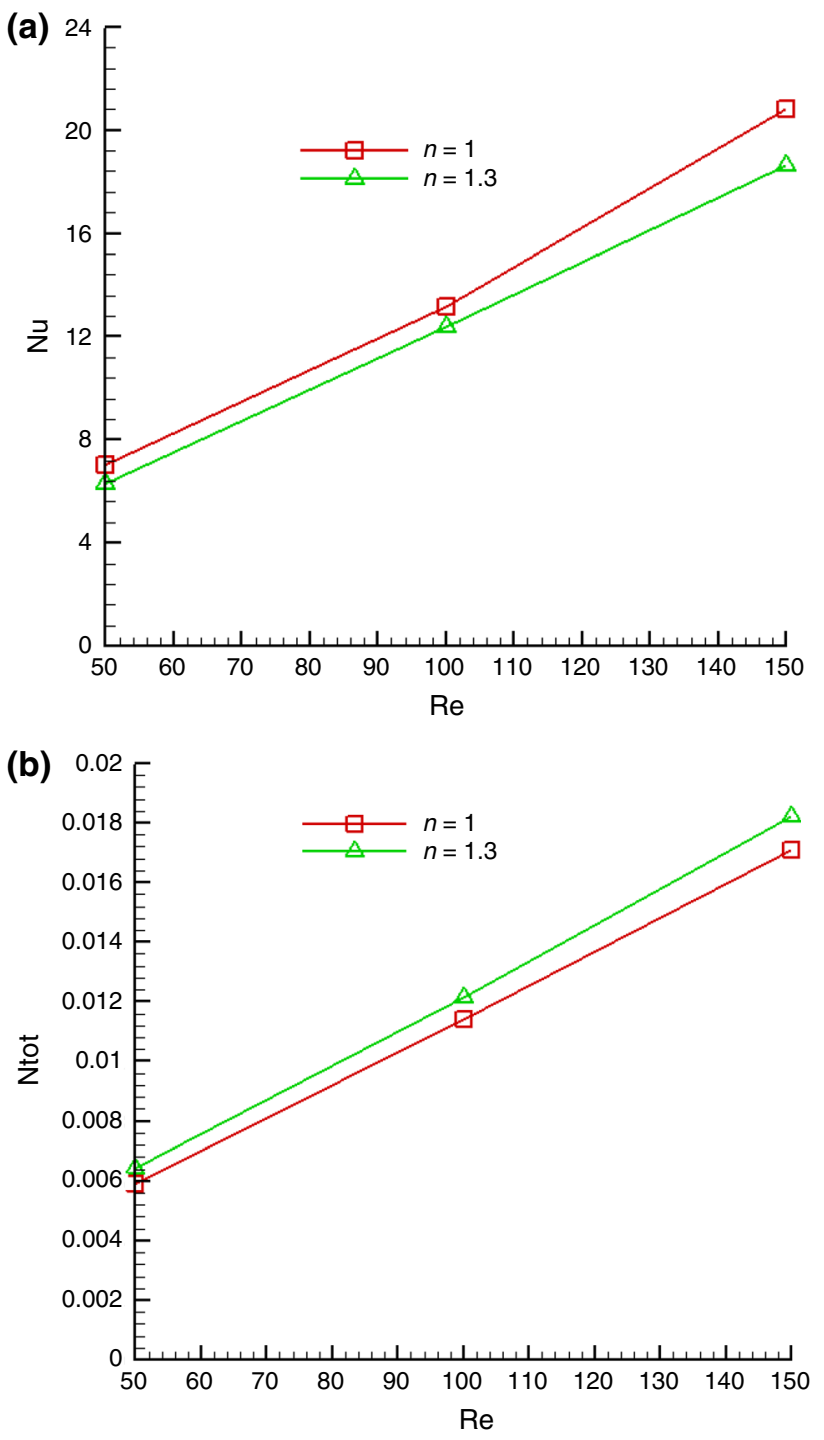

Fig. 13 a Nusselt number and b Total entropy generation versus the Reynolds number for different values of non-Newtonian fluid $(n=1.3)$ and Newtonian fluid $(n=0.5)$ indices

\section{Conclusions}

Transport of heat and mass and thermodynamic irreversibilities in a parallel-plate microreactor filled by a homogenous porous medium and with a non-Newtonian working fluid were investigated. The considered configuration was assumed to be geometrically and thermally asymmetric. The power-law behavior was assumed for the fluid, and the porous medium was set under local thermal non-equilibrium. A set of governing equations were first derived theoretically. The resultant nonlinear system of partial differential equations was then solved numerically through using a finite volume solver. This led to the development of two-dimensional solutions for the temperature concentration and local entropy generation fields and evaluation of Nusselt number and total entropy generation. The numerical results were validated against the existing fully analytical solutions for the Newtonian fluids. It was shown that increases in the value of power-law index reduce the rate of heat transfer and thus drop the value of Nusselt number. Hence, the local and total generations of entropy are both strongly affected by the value of power-law index in which increases in this parameter highly enhance the local and total irreversibilities. The other qualitative characteristics of the investigated system were found to remain consistent with those reported for the Newtonian fluids.

Open Access This article is licensed under a Creative Commons Attribution 4.0 International License, which permits use, sharing, adaptation, distribution and reproduction in any medium or format, as long as you give appropriate credit to the original author(s) and the source, provide a link to the Creative Commons licence, and indicate if changes were made. The images or other third party material in this article are included in the article's Creative Commons licence, unless indicated otherwise in a credit line to the material. If material is not included in the article's Creative Commons licence and your intended use is not permitted by statutory regulation or exceeds the permitted use, you will need to obtain permission directly from the copyright holder. To view a copy of this licence, visit http://creativecommons.org/licenses/by/4.0/.

\section{References}

1. Hunt G, Karimi N, Torabi M. Two-dimensional analytical investigation of coupled heat and mass transfer and entropy generation in a porous, catalytic microreactor. Int J Heat Mass Transf. 2018;119:372-91.

2. Saeed A, Karimi N, Hunt G, Torabi M. On the influences of surface heat release and thermal radiation upon transport in catalytic porous microreactors-a novel porous-solid interface model. Chem Eng Process. 2019;143:107602. https://doi.org/10.1016/j. cep.2019.107602.

3. Hunt G, Karimi N, Yadollahi B, Torabi M. The effects of exothermic catalytic reactions upon combined transport of heat and mass in porous microreactors. Int J Heat Mass Transf. 2019;134:122749. https://doi.org/10.1016/j.ijheatmasstransfer.2019.02.015.

4. Alizadeh R, Karimi N, Mehdizadeh A, Nourbakhsh A. Analysis of transport from cylindrical surfaces subject to catalytic reactions and non-uniform impinging flows in porous media- A nonequilibrium thermodynamics approach. J Therm Anal Calorim. 2019;138:659-78. https://doi.org/10.1007/s10973-019-08120-z.

5. Torabi M, Karimi N, Peterson GP, Yee S. Challenges and progress on the modelling of entropy generation in porous media: a review. Int J Heat Mass Transf. 2017;114:31-46. https://doi.org/10.1016/j. ijheatmasstransfer.2017.06.021.

6. SacitHerdem M, Mundhwa M, Farhad S, Hamdullahpur F. Catalyst layer design and arrangement to improve the performance of a microchannel methanol steam reformer. Energy Convers Manage. 2019;180:149-61.

7. Hunt G, Torabi M, Govone L, Karimi N, Mehdizadeh A. Two-dimensional heat and mass transfer and thermodynamic analyses of porous microreactors with Soret and thermal radiation effects-an analytical approach. Chem Eng Process Process Intensif. 2018;126:190-205. https://doi.org/10.1016/j. cep.2018.02.025. 
8. Mottaghi M, Kuhn S. Numerical investigation of well-structured porous media in a milli-scale tubular reactor. Chem Eng Sci. 2019;208:115146. https://doi.org/10.1016/j.ces.2019.08.004.

9. Liu Y, Zhou W, Chen L, Lin Y, Xuyang C, Zheng T, Wan S. Optimal design and fabrication of surface microchannels on copper foam catalyst support in a methanol steam reforming microreactor. Fuel. 2019;253:1545-55. https://doi. org/10.1016/j.fuel.2019.05.099.

10. Ambrosetti M, Bracconi M, Maestri M, Groppi G, Tronconi E. Packed foams for the intensification of catalytic processes: assessment of packing efficiency and pressure drop using a combined and numerical approach. Chem Eng J. 2020;382:122801. https://doi.org/10.1016/j.cej.2019.122801.

11. Lee D, SeokKim B, Moon H, Lee N, Shin S, HeeCho H. Enhanced boiling heat transfer on nanowire-forested surfaces under subcooling conditions. Int J Heat Mass Transf. 2018;120:1020-30. https ://doi.org/10.1016/j.ijheatmasstransfer.2017.12.100.

12. Li Y, Zhao M, Li C, Ge W. Concentration fluctuation due to reaction-diffusion coupling near an isolated active site on catalyst surfaces. Chem Eng J. 2019;373:744-54. https://doi.org/10.1016/j. cej.2019.05.052.

13. Li J, An H, Sasmito AP, Mujumdar AS, Ling X. Performance evaluation of mass transport enhancement in novel dual-channel design of micro-reactors. Heat Mass Transf. 2019;56:559-74.

14. Rossetti I. Continuous flow (micro-) reactors for heterogeneously catalyzed reactions: main design and modelling issues. Catal Today. 2018;308:20-31.

15. Roychowdhury S, Sundararajan T, Das SK. Conjugate heat transfer studies on steam reforming of ethanol in micro-channel systems. Int J Heat Mass Transf. 2019;139:660-74.

16. Hosseini SR, Ghasemian M, Sheikholeslami M, Shafee A, Li Z. Entropy analysis of nanofluid convection in a heated porous microchannel under MHD field considering solid heat generation. Powder Technol. 2019;344:914-25.

17. Gireesha BJ, Srinivasa CT, Shashikumar NS, Macha M, Singh JK, Mahanthesh B. Entropy generation and heat transport analysis of Casson fluid flow with viscous and Joule heating in an inclined porous microchannel. Proc IME E J Process Mech Eng. 2019;233:1173-84.

18. Ranjit NK, Shit GC. Entropy generation on electromagnetohydrodynamic flow through a porous asymmetric micro-channel. Eur J Mech B Fluids. 2019;77:135-47.

19. Mahmoudi Y, Hooman K, Vafai K. Convective heat transfer in porous media.] 2019. https://doi.org/10.1201/9780429020261.

20. Sajadifar SA, Karimipour A, Toghraie D. Fluid flow and heat transfer of non-Newtonian nanofluid in a microtube considering slip velocity and temperature jump boundary conditions. Eur J Mech B Fluids. 2017;61:25-32.

21. Fu T, Wei L, Zhu C, Ma Y. Flow patterns of liquid-liquid twophase flow in non-Newtonian fluids in rectangular microchannels. Chem Eng Process. 2015;91:114-20.

22. Neffah Z, Kahalerras H, Fersadou B. Heat and mass transfer of a non-newtonian fluid flow in an anisotropic porous channel with chemical surface reaction. FDMP. 2018;14:39-56. https://doi. org/10.3970/fdmp.2018.014.039.

23. Wang Z, Wei Y, Qian Y. Numerical study on entropy generation in thermal convection with differentially discrete heat boundary conditions. Entropy. 2018;20:351. https://doi.org/10.3390/ e20050351.

24. Saeed A, Karimi N, Hunt G, Torabi M, Mehdizadeh A. Doublediffusive transport and thermodynamic analysis of a magnetic microreactor with non-Newtonian biofuel flow. J Therm Anal Calorim. 2019. https://doi.org/10.1007/s10973-019-08629-3.
25. Gholamalizadeh E, Pahlevanzadeh F, Ghani K, Karimipour A, Nguyen TK, Safaei. Simulation of water/FMWCNT nanofluid forced convection in a microchannel filled with porous material under slip velocity and temperature jump boundary Conditions. Int J Numer Methods Heat Fluid Flow. 2019. https://doi. org/10.1108/HFF-01-2019-0030.

26. Maleki H, Safaei MR, Alrashed AA, Kasaeian A. Flow and heat transfer in non-Newtonian nanofluids over porous Surfaces. J Therm Anal Calorim. 2019;135:1655-66. https://doi. org/10.1007/s10973-018-7277-9.

27. Animasaun IL, Pop I. Numerical exploration of a non-Newtonian Carreau fluid flow driven by catalytic surface reactions on an upper horizontal surface of a paraboloid of revolution, buoyancy and stretching at the free stream. Alexandria Eng J. 2017;56:647-58. https://doi.org/10.1016/j.aej.2017.07.005.

28. Al-Rashed AA, Shahsavar A, Entezari S, Moghimi MA, Adio SA, Nguyen TK. Numerical investigation of non-Newtonian water-CMC/CuO nanofluid flow in an offset strip-fin microchannel heat sink: thermal performance and thermodynamic considerations. Appl Therm Eng. 2019;155:247-58. https://doi. org/10.1016/j.applthermaleng.2019.04.009.

29. Kiyasatfar M. Convective heat transfer and entropy generation analysis of non-Newtonian power-law fluid flows in parallel-plate and circular microchannels under slip boundary conditions. Int J Therm Sci. 2018;128:15-27. https://doi. org/10.1016/j.ijthermalsci.2018.02.013.

30. Gheynani AR, Akbari OA, Zarringhalam M, Shabani GAS, Alnaqi AA, Goodarzi M, Toghraie D. Investigating the effect of nanoparticles diameter on turbulent flow and heat transfer properties of non-Newtonian carboxymethyl cellulose/CuO fluid in a microtube. Int J Numer Methods Heat Fluid Flow. 2018. https://doi.org/10.1108/HFF-07-2018-0368.

31. Mukherjee S, Prayag B, Suman C, Sunando D. Effects of viscous dissipation during forced convection of power-law fluids in microchannels. Int Commun Heat Mass Transf. 2017;89:83-90.

32. Torabi M, Elliott A, Karimi N. Thermodynamics analyses of porous microchannels with asymmetric thick walls and exothermicity: an entropic model of micro-reactors. J Therm Sci Eng. 2017;9:041013. https://doi.org/10.1115/1.4036802.

33. Torabi M, Torabi M, Peterson GP. Entropy generation of double diffusive forced convection in porous channels with thick walls and Soret effect. Entropy. 2017;19:171. https://doi.org/10.3390/ e19040171.

34. Elliott A, Torabi M, Karimi N. Thermodynamics analyses of porous microchannels with asymmetric thick walls and exothermicity: an entropic model of microreactors. J Therm Sci Eng 2017;9:041013.https://doi.org/10.1115/1.4036802.

35. Karimi N, Agbo D, Khan AT, Younger PL. On the effects of exothermicity and endothermicity upon the temperature fields in a partially-filled porous channel. Int J Therm Sci. 2015;96:128-48.

36. Guthrie DGP, Torabi M, Karimi N. Combined heat and mass transfer analyses in catalytic microreactors partially filled with porous material-The influences of nanofluid and different porousfluid interface models. Int J Thermal Sci. 2019;140:96-113. https ://doi.org/10.1016/j.jithermalsci.2019.02.037.

37. Athar K, Doranehgard MH, Eghbali S, Dehghanpour H. Measuring diffusion coefficients of gaseous propane in heavy oil at elevated temperatures. J Therm Anal Calorim. 2020;139:2633-45.

38. Saffarian MR, Moravej M, Doranehgard MH. Heat transfer enhancement in a flat plate solar collector with different flow path shapes using nanofluid. Renew Energy. 2020;146:2316-29. 
39. Bozorg MV, Doranehgard MH, Hong K, Xiong Q, Li LK. A numerical study on discrete combustion of polydisperse magnesium aero-suspensions. Energy. 2020;194:116872.

40. Nabipour N, Daneshfar R, Rezvanjou O, Mohammadi-Khanaposhtani M, Baghban A, Xiong Q, Doranehgard MH. Estimating biofuel density via a soft computing approach based on intermolecular interactions. Renew Energy. 2020;152:1086-98.
41. Habib R, Karimi N, Yadollahi B, Doranehgard MH, Li LK. A pore-scale assessment of the dynamic response of forced convection in porous media to inlet flow modulations. Int J Heat Mass Transf. 2020;153:119657.

Publisher's Note Springer Nature remains neutral with regard to jurisdictional claims in published maps and institutional affiliations. 